Review Article

\title{
Bioactive Substances in Safflower Flowers and Their Applicability in Medicine and Health-Promoting Foods
}

\author{
I. Adamska $(\mathbb{D})$ and P. Biernacka \\ Department of Fish, Plant and Gastronomy Technology, West Pomeranian University of Technology in Szczecin, Szczecin, Poland \\ Correspondence should be addressed to I. Adamska; iwona.adamska@zut.edu.pl
}

Received 4 December 2020; Revised 15 April 2021; Accepted 5 May 2021; Published 27 May 2021

Academic Editor: Eduard Hernández

Copyright ( 2021 I. Adamska and P. Biernacka. This is an open access article distributed under the Creative Commons Attribution License, which permits unrestricted use, distribution, and reproduction in any medium, provided the original work is properly cited.

\begin{abstract}
Safflower flowers (Carthamus tinctorius) contain many natural substances with a wide range of economic uses. The most famous dye isolated from flower petals is hydroxysafflor A (HSYA), which has antibacterial, anti-inflammatory, and antioxidant properties. This review is aimed at updating the state of knowledge about their applicability in oncology, pulmonology, cardiology, gynecology, dermatology, gastrology, immunology, and suitability in the treatment of obesity and diabetes and its consequences with information published mainly in 2018-2020. They were also effective in treating obesity and diabetes and its consequences. The issues related to the possibilities of using HSYA in the production of health-promoting food were also analyzed.
\end{abstract}

\section{Introduction}

The demand for natural compounds used in the key branches of economy is increasing in connection with the growing awareness of consumers. However, producers are forced to follow economic considerations and use cheaper equivalents of certain ingredients. For this reason, safflower (Carthamus tinctorius), treated as a low-cost replacement for saffron crocus (Crocus sativus L.) and as a rich source of bioactive substances, has been applied in numerous branches of economy.

Saffron is known for its rich chemical composition: simple and complex sugars, amino acids, proteins, lipids, cellulose, mineral compounds, and vitamins, including thiamine and riboflavin, low amounts of $\alpha$-carotene and $\beta$-carotene, zeaxantin, and lycopene. However, the content and bioactivity of several glycosides in flowers (safranal, picrocrocin, crocetin, and crocins) are an indicator of the attractiveness of this plant [1-7]. These glycosides have not yet been found in safflower flowers.

The difference in the price of saffron and safflower as an industrial raw material depends on the crop yield. Commercial saffron (spice) accounts for only $8 \%$ of the total flower yield. One plant produces a maximum of 9 pistil stigmas (up to 3 flowers, each with 3 stigmas) [8]. The average weight of the flower pistil is about $2 \mathrm{mg}$, so a maximum of about $18 \mathrm{mg}$ of raw material is obtained from one plant. It has been estimated that one kilogram of spice is made from 150,000 flowers [9]. In the case of safflower, the raw material is the whole flowers (petals with stamens) formed in large inflorescences (each contains 20-250 flowers [10]).

The purpose of the study is to review the current information (mainly published in 2018-2020) on the chemical composition of the substances present in the flowers and green parts of safflower (Carthamus tinctorius), their impact on humans, and the possibility of using them in the production of health-promoting food. For the first time, the possibilities of using hydroxysafflor $\mathrm{A}$, the main bioactive component, were analyzed.

\section{Botanical Characteristics and Cultivation Requirements}

Safflower (Carthamus tinctorius) is classified in the Asteraceae family. Its natural area of distribution covers Asia (area of India) and Middle East. The plant has bushy habit and it reaches the height of $100-130 \mathrm{~cm}$. It forms large, lanceolate leaves with serrated margins. Safflower flowers are radial and tubular and they form large inflorescences (flower heads) 
[10-12]. Four cultivar groups were distinguished depending on the color of the flowers before and after drying: (1) yellow blooming and red dried flowers, (2) yellow blooming and dried flowers, (3) orange blooming and dark red dried flowers, and (4) white blooming and dried flowers [10].

Safflower is resistant to wind, drought [13], and salinity [14]. It can be cultivated on low-fertility soils (Koutroubas and Papakosta (2005), after [15]), preferably in warm and not too humid climate. The largest plantations are located in India and Bengal, southern France, USA, Iran, Egypt, and China $[13,16,17]$.

The quality of raw material obtained from safflower depends on the conditions prevailing in the cultivation area: air temperature and humidity, soil moisture, insolation, and soil fertility $[15,18-22]$. The intensity of insolation affects the content of flavonoids: their synthesis was higher under the conditions of limited exposure of plants to sunlight [22]. The features of the cultivated plant are also important $[15,23-25]$. Studies are currently conducted aiming at obtaining safflower cultivars with the highest possible content of pigments in flower petals [26].

\section{Chemical Composition of Safflower Green Parts and Flowers}

The above-ground parts exhibit high content of carbon (42.7-49.1\% d.w.) and relatively low nitrogen (0.36-1.23\%) [27]. They contain carthamusin A [17], $\beta$-daucosterol, and stigmasterol [28].

The chemical composition of flowers is interesting and rich (Table 1), with 200 substances identified thus far [29]. Flower petals contain $1.82 \%$ protein, $4.8 \%$ lipids, $11.6 \%$ crude fiber, and $10.8 \%$ ash, and their moisture content is $4.7 \%$ [30]. They also contain, among others, alkaloids, flavonoids, lignanoids, organic acids and polyacetylenes [17, 31], alkanediols, riboflavin, steroids, and quinochalcone C-glycosides [29].

Most of the pigments found in flower petals are flavonoids of the C-glucosylquinochalcone group. The best known are carthamine (also known as safflower yellow, carthamus red, or carthamine) and carthamidin (synonyms include carthamic acid). Carthamine $\left(\mathrm{C}_{43} \mathrm{H}_{42} \mathrm{O}_{22}\right)$, red pigment, is flavonoid compound consisting of two chalkonoids. It is formed as a result of oxidation with precarthamine. It is insoluble in water and it usually constitutes $3-6 \%$ of petal composition; however, in some flower parts, the content is below 1\% [30]. During the biosynthesis of carthamine flowers, color gradually changes from yellow to red [32]. The yellow pigment, carthamidin, constitutes $24-30 \%$ of compounds found in the flowers of safflower. It is a tetrahydroxyflavanone, (S)-naringenin derivative, water-soluble substance [30]. The following flavonoids have been identified: hydroxysafflor yellow A (HSYA; also known as safflomin A), 6-hydroxykaempferol 3,6-di- $O-\beta$ d-glucoside-7-O- $\beta$-d-glucuronide, 6-hydroxykaempferol 3,6,7tri- $O$ - $\beta$-d-glucoside, 6-hydroxykaempferol 3-O- $\beta$-rutinoside6-O- $\beta$-d-glucoside, 6-hydroxykaempferol 3,6-di- $O$ - $\beta$-d-glucoside, 6-hydroxyapigenin 6-O-glucoside-7-O-glucuronide, anhydrosafflor yellow $\mathrm{B}$, kaempferol 3-O- $\beta$-rutinoside, and two compounds from other groups: guanosine and syringin [33]. In the course of further study, the following substances were also isolated: (2S)- $4^{\prime}, 5$-dihydroxyl-6,7-di-O- $\beta$-D-glucopyranosyl flavanone, 6-hydroxy kaempferol 3-O- $\beta$-D-glucoside, 6hydroxykaempferol 3-O- $\beta$-D-rutinoside, 6-hydroxykaempferol 6,7-di-O- $\beta$-D-glucoside, and 6-hydroxyquercetin 3,6,7-tri-O$\beta$-D-glucoside [34]. Other ingredients isolated from aqueous extracts of flower petals were quinochalcones (cartormin, isosafflomin C, precarthamine, safflomin B, safflomin C, safflor yellow A, safflor yellow B, and tinctormin [35]) and flavonoids (azaleatin $\left(3,7,3^{\prime}, 4^{\prime}\right.$-tetrahydroxy-5-methoxyflavone), saffloroside $\left(3,7,3^{\prime}, 4^{\prime}\right.$-tetrahydroxy-5-methoxyflavone 7 -O- $\beta$-D-glucopyranoside), 5-O-methylluteolin $[36,37]$, cinaroside $\left(5,7,3^{\prime}, 4^{\prime}\right.$ -tetrahydroxyflavone 7-O- $\beta$-D-glucopyranoside) [36], and 6hydroxykaempferol-3-O- $\beta$-D-glucoside-7-O- $\beta$-D-glucuronide [38]).

Aqueous extract of flowers also contained isocartormin, new semiquinonechalcone C-glycoside, which is a cartormin isomer [29], while from immature, yellow petals of C. tinctorius-precarthamine, the yellow precursor of carthamine. Unripe flower petals contain an enzyme that converts precarthamine into carthamine [32].

There is a correlation between the content of active substances and the intensity of flower's color. Among the active substances, hydroxysafflor A has always been predominant (independently of the degree in which flowers are colored); however, flowers with more intense coloration (vivid red and bright yellow or bright orange) were characterized by higher content of HSYA, anhydrosafflor yellow B, kaempferol, quercetin, safflomin C, kaempferol-3-O-rutinoside, and 6-hydroxykaempferol-3-0- $\beta$-d-glucoside compared with less colored flowers [39, 40].

3.1. Essential Oils. Studies showed the presence of 20 [41] and 29 [42] substances included in the safflower essential oil. The highest content in the tests [41] characterized by heptacosane (34.8\%), nonanoic acid (17.9\%), and dec-2-en-1-ol (14.3\%) and in study [42] 1-hydroxy-3-propyl-5-(4-methyl-penten)2-methylbenzene (25.2\%) and 2,5,5 trimethyl 3-n propyl, tetra hydro1-naphtol (19.8\%) were predominant.

\section{Applicability of Safflower Bioactive Substances in Medicine}

Substances isolated from safflower flowers have been used in medicine (summarized in Figure 1). Water-soluble components are particularly important in this case, especially intravenously administered quinochalcone C-glycosides [34]. Safflower is attributed with analgesic, anti-inflammatory, and antiaging properties (after [17]). Most studies have been devoted to the use of hydroxysafflor A (HSYA); however, positive impact of yellow carthamine (CY), safflor A (SA), safflower yellow (SY), hydroxysafflor B (HSYB), hydroxysafflor C (HSYC), and selected water-soluble polysaccharides on human health has been also demonstrated (summarized in Tables 2-5).

4.1. Antioxidative, Antiseptic, and Anti-Inflammatory Effect. Hydroxysafflor yellow A has an antioxidant effect, enabling cells to survive oxidative stress [43-50]. Antioxidant activity is also demonstrated by hydroxysafflor yellow B [51], 
TABLE 1: The main components of the safflower flower extract.

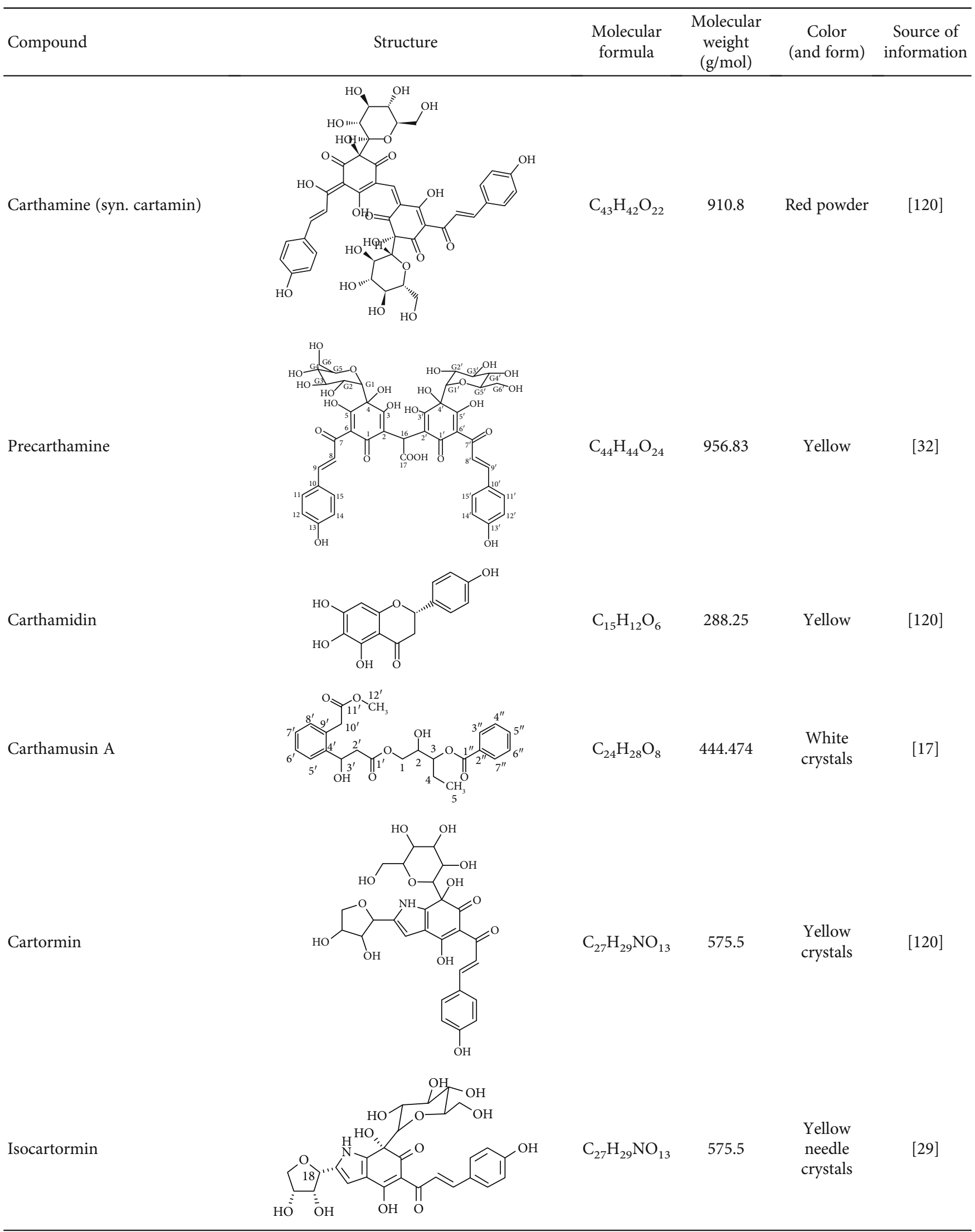


TABle 1: Continued.

\begin{tabular}{|c|c|c|c|c|c|}
\hline Compound & Structure & $\begin{array}{l}\text { Molecular } \\
\text { formula }\end{array}$ & $\begin{array}{l}\text { Molecular } \\
\text { weight } \\
(\mathrm{g} / \mathrm{mol})\end{array}$ & $\begin{array}{c}\text { Color } \\
\text { (and form) }\end{array}$ & $\begin{array}{c}\text { Source of } \\
\text { information }\end{array}$ \\
\hline $\begin{array}{l}\text { Hydroxysafflor yellow A } \\
\text { (HSYA) }\end{array}$ & & $\mathrm{C}_{27} \mathrm{H}_{32} \mathrm{O}_{16}$ & 612.5 & Yellow & {$[120]$} \\
\hline $\begin{array}{l}\text { Anhydrosafflor yellow B } \\
\text { (AHSYB) }\end{array}$ & & $\mathrm{C}_{48} \mathrm{H}_{52} \mathrm{O}_{26}$ & 1044.9 & Yellow & [120] \\
\hline Safflomin C & & $\mathrm{C}_{30} \mathrm{H}_{30} \mathrm{O}_{14}$ & 614.5 & $\begin{array}{l}\text { Yellow } \\
\text { powder }\end{array}$ & {$[121]$} \\
\hline Isosafflomin C & & $\mathrm{C}_{30} \mathrm{H}_{30} \mathrm{O}_{14}$ & 614.5 & Yellow & {$[122]$} \\
\hline Safflor yellow A & & $\mathrm{C}_{27} \mathrm{H}_{30} \mathrm{O}_{16}$ & 610.5 & Yellow & {$[120]$} \\
\hline
\end{tabular}


TABle 1: Continued.

\begin{tabular}{|c|c|c|c|c|c|}
\hline Compound & Structure & $\begin{array}{l}\text { Molecular } \\
\text { formula }\end{array}$ & $\begin{array}{c}\text { Molecular } \\
\text { weight } \\
(\mathrm{g} / \mathrm{mol})\end{array}$ & $\begin{array}{c}\text { Color } \\
\text { (and form) }\end{array}$ & $\begin{array}{c}\text { Source of } \\
\text { information }\end{array}$ \\
\hline Safflor yellow B (syn. safflomin B) & & $\mathrm{C}_{48} \mathrm{H}_{54} \mathrm{O}_{27}$ & 1062.9 & Yellow & [120] \\
\hline Saffloroside & & $\mathrm{C}_{22} \mathrm{H}_{22} \mathrm{O}_{12}$ & 478.403 & $\begin{array}{l}\text { Yellow } \\
\text { crystals }\end{array}$ & {$[37]$} \\
\hline Azaleatin & & $\mathrm{C}_{16} \mathrm{H}_{12} \mathrm{O}_{7}$ & 316.26 & $\begin{array}{l}\text { Yellow } \\
\text { crystals }\end{array}$ & {$[120]$} \\
\hline Cinaroside & & $\mathrm{C}_{21} \mathrm{H}_{20} \mathrm{O}_{11}$ & 448.377 & $\begin{array}{l}\text { Light-yellow } \\
\text { crystals }\end{array}$ & {$[120]$} \\
\hline Guanosine & & $\mathrm{C}_{10} \mathrm{H}_{13} \mathrm{~N}_{5} \mathrm{O}_{5}$ & 283.24 & $\begin{array}{l}\text { White } \\
\text { crystalline } \\
\text { powder }\end{array}$ & {$[120]$} \\
\hline Kaempferol & & $\mathrm{C}_{15} \mathrm{H}_{10} \mathrm{O}_{6}$ & 286.24 & $\begin{array}{l}\text { Light yellow } \\
\text { powder }\end{array}$ & {$[120]$} \\
\hline
\end{tabular}


TABle 1: Continued.

\begin{tabular}{|c|c|c|c|c|c|}
\hline Compound & Structure & $\begin{array}{l}\text { Molecular } \\
\text { formula }\end{array}$ & $\begin{array}{l}\text { Molecular } \\
\text { weight } \\
(\mathrm{g} / \mathrm{mol})\end{array}$ & $\begin{array}{c}\text { Color } \\
\text { (and form) }\end{array}$ & $\begin{array}{l}\text { Source of } \\
\text { information }\end{array}$ \\
\hline $\begin{array}{l}\text { Kaempferol 3-O- } \beta \text {-rutinoside } \\
\text { (syn. nicotiflorin) }\end{array}$ & & $\mathrm{C}_{27} \mathrm{H}_{30} \mathrm{O}_{15}$ & 594.5 & $\begin{array}{l}\text { Yellow, } \\
\text { powder or } \\
\text { crystals }\end{array}$ & [121] \\
\hline Luteolin & & $\mathrm{C}_{15} \mathrm{H}_{10} \mathrm{O}_{6}$ & 286.2 & $\begin{array}{l}\text { Yellow } \\
\text { crystals }\end{array}$ & [120] \\
\hline $\begin{array}{l}\text { Luteolin 5-methyl ether } \\
\text { (syn. 5-O-methylluteolin) }\end{array}$ & & $\mathrm{C}_{16} \mathrm{H}_{12} \mathrm{O}_{6}$ & 300.3 & $\begin{array}{l}\text { Yellow } \\
\text { powder }\end{array}$ & [120] \\
\hline Quercetin & & $\mathrm{C}_{15} \mathrm{H}_{10} \mathrm{O}_{7}$ & 302.2 & $\begin{array}{l}\text { Yellow } \\
\text { needle } \\
\text { crystals }\end{array}$ & [121] \\
\hline Stigmasterol & & $\mathrm{C}_{29} \mathrm{H}_{48} \mathrm{O}$ & 412.7 & $\begin{array}{l}\text { White } \\
\text { powder }\end{array}$ & [120] \\
\hline Syringin & & $\mathrm{C}_{17} \mathrm{H}_{24} \mathrm{O}_{9}$ & 372.4 & $\begin{array}{l}\text { White } \\
\text { crystals }\end{array}$ & [120] \\
\hline
\end{tabular}


TABLE 1: Continued.

\begin{tabular}{|c|c|c|c|c|c|}
\hline Compound & Structure & $\begin{array}{l}\text { Molecular } \\
\text { formula }\end{array}$ & $\begin{array}{c}\text { Molecular } \\
\text { weight } \\
(\mathrm{g} / \mathrm{mol})\end{array}$ & $\begin{array}{c}\text { Color } \\
\text { (and form) }\end{array}$ & $\begin{array}{l}\text { Source of } \\
\text { information }\end{array}$ \\
\hline Tinctormine & & $\mathrm{C}_{27} \mathrm{H}_{31} \mathrm{NO}_{14}$ & 593.5 & $\begin{array}{l}\text { Yellow } \\
\text { powder }\end{array}$ & {$[121]$} \\
\hline$\beta$-Daucosterol (syn. sitogluside) & & $\mathrm{C}_{35} \mathrm{H}_{60} \mathrm{O}_{6}$ & 576.8 & $\begin{array}{l}\text { White } \\
\text { powder }\end{array}$ & {$[121]$} \\
\hline $\begin{array}{l}\text { 6-Hydroxykaempferol } \\
\text { 3-O- } \beta \text {-D-rutinoside }\end{array}$ & & $\mathrm{C}_{27} \mathrm{H}_{30} \mathrm{O}_{16}$ & 610.5 & $\begin{array}{l}\text { Yellow } \\
\text { powder }\end{array}$ & {$[121]$} \\
\hline $\begin{array}{l}\text { 6-Hydroxykaempferol-3-O- } \beta \text { - } \\
\text { D-glucoside-7-O- } \beta \text {-D- } \\
\text { glucuronide }\end{array}$ & & $\mathrm{C}_{27} \mathrm{H}_{28} \mathrm{O}_{17}$ & 624.5 & Yellow & [38] \\
\hline
\end{tabular}

hydroxysafflor yellow C [52], safflor A (SYA) [48], and carthamine [53]. However, safflower flower extract as well as HSYA and SYA acted in this manner only at low concentrations, whereas in higher concentrations they exhibited prooxidative effect favoring creation of reactive oxygen species in the cells [47]. The paste produced from crushed safflower leaves accelerated the healing of difficult to control wounds (Dehariya et al. (2015), after [54]). Another representative of the genus Carthamus (C. oxyacantha) also shows antibacterial and antidiarrheal effect. Substances contained in the plant limited the proliferation of Escherichia, Pseudomonas, Salmonella, and Staphylococcus [55]. Moschamine obtained from $C$. tinctorius seeds has anti-inflammatory effect: it inhibits prostaglandin E2 and nitric oxide production in macrophages [56].

4.2. Immune System. Products containing HSYA have enabled preventing acute anaphylaxis in mice. Anaphylaxis may appear in response to contact with allergen or drug, and it is associated with a rapid activation of mediators (i.e., tumor necrosis factor, histamine, $\beta$-hexosaminidase, or monocyte chemotactic protein from mast cells), which may result in death. Administration of HSYA has clearly inhibited degranulation of mast cells via impeding $\mathrm{Ca}^{2+}$ transport and the release of cytokines and chemokines [57].

4.3. Hormone and Reproductive System. HSYA injection led to reduction of cysts on mouse ovaries with polycystic ovary syndrome, and at the same time, it regulated the hormonal balance and restored normal ovulation cycle by reducing the levels of testosterone and follicle-stimulating hormone (FSH) in the blood and increasing the level of progesterone, estradiol, and luteinizing hormone [46]. Anti-Müllerian hormone level, an indicator of the ovulation process, also increased.

4.4. Diabetes-Related Complications. Untreated diabetes results in increased level of advanced glycation end products 


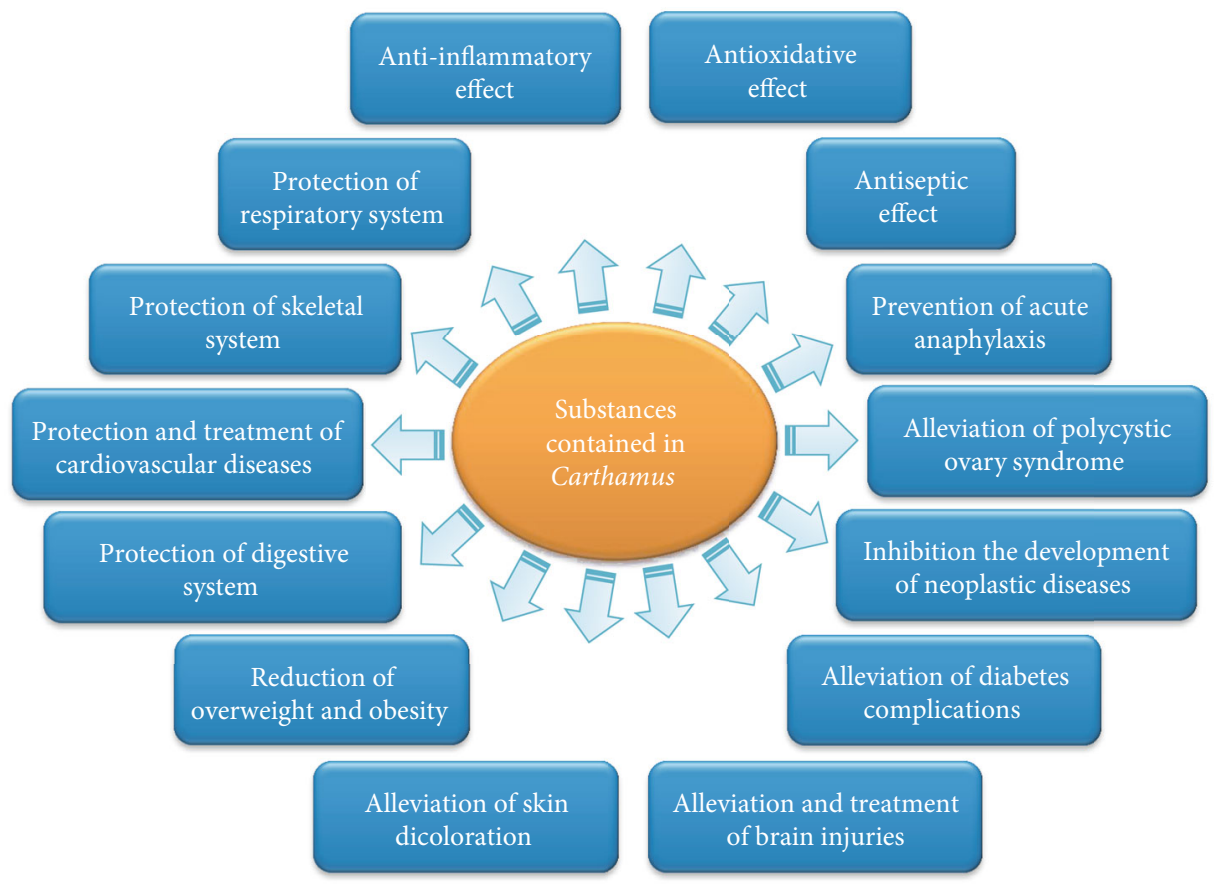

FIGURE 1: Bioactive effect of the safflower substances.

(AGEs) and methylglyoxal in the organism's cells, which have toxic effect on cells (apoptosis), tissues (accelerated aging), and organs (destruction). In the course of diabetes, increased content of caspase-3 is observed, which is responsible for, among others, degradation of the ICAD protein (inhibitor of caspase-activated DNase), DAFF40/CAD endonuclease inhibitor. This leads to activation of endonuclease and DNA fragmentation. Lower amounts of AGEs are formed in the organism after administration of HSYA, which impedes cell apoptosis. This compound has protective effect on the microvascular endothelium in the human brain, which reduces the range of damage caused by elevated AGE level [58].

Furthermore, hydroxysafflor A has protective effect towards pancreatic cells. High blood glucose concentration leads to oxidative damage and apoptosis of $\beta$ cells in the pancreas, leading to its disturbed function. HSYA reduces oxidative injury of cells and results in inhibited apoptosis of $\beta$ cells via blocking the JNK/c-Jun signaling pathway [44] and affecting the course of the PI3K-AKT signaling pathway (the phosphatidylinositol 3-kinase and AKT protein kinase pathway) [59]. HSYA reduces the parameters enabling the assessment of the level of oxidative stress (levels of catalase, glutathione peroxidase, lipid peroxidation, reactive oxygen species, and superoxide dismutase) and indicators of pancreas cell apoptosis level (content of caspase-3 and parp) [44]. HSYA administration resulted in reduced level of fasting glucose in the blood of rats and reduced insulin resistance, as well as positive effect on lipid metabolism. This decreased the total and low-density lipoprotein cholesterol and triglyceride levels, and the level of glycogen synthase and hepatic glycogen was increased [59].

Favorable impact of HSYA was also observed in the treatment of kidney fibrosis associated with diabetes in rats. This substance affected the course of TLR4/NF- $\kappa \mathrm{B} / \mathrm{p} 65$ signaling pathway and produced improvement (reduction) of a series of tested parameters (including miRNA-140-5p mRNA, $24 \mathrm{~h}$ UP, TC, or TNF- $\alpha$ and the level of proteins: Col-IV, NF- $\kappa \mathrm{B}(\mathrm{p} 65)$, NLRP3, Notch2, and TLR4) and relieving the symptoms of kidney fibrosis [60].

Administration of methanol extract of safflower flowers containing phenolic compounds (i.e., acids: caffeic, catechin, catechol, chlorogenic, ellagic, protocatechuic, and vanillic) and flavonoids (i.e., hesperidin, kaempferol, narengin, quercetin, quercitrin, rosmarinic, rutin, and 7-hydroxyflavone) reduced symptoms of pancreas dysfunction in rats with diabetes. This extract exhibited high reducing force and antioxidative activity $\left(\mathrm{DPPH}^{+}\right)[49]$.

4.5. Nervous System. As shown for rats, HSYA reduces the effects of brain injuries (contusions). It inhibited development of symptoms, favored the increase of superoxide dismutase and ATPase activity, increased the amount of tissue plasminogen activator, and resulted in reduced level of plasminogen-1 activator inhibitor in the blood plasma as well as malondialdehyde in the tissues adjacent to the injury [61]. Furthermore, HSYA is efficient in treatment of brain ischemic injuries $[62,63]$, as it inhibits activation of the pyroptosis pathway (brain cell death induced by caspase-1 resulting from nerve damage). HSYA promotes the inhibition of cell apoptosis process and enhances viability of the cells damaged as a result of oxidative stress. It is also a part of the Lex-HSYA complex which activates injury relieving factors, and this restricts the range of cerebral infarction [63]. What is more, administration of HSYA resulted in alleviation and decreasing the rate of proinflammatory and oxidative reactions in the tissues involved by ischemia-reperfusion injury of rat brain [64] and induced vasodilation of cerebral and 
TABLE 2: Selected studies of antioxidant activity of substances present in safflower flowers.

\begin{tabular}{|c|c|c|c|c|c|c|}
\hline Substance & Research object & Type of test & $\begin{array}{l}\text { Parameter } \\
\text { determined }\end{array}$ & $\begin{array}{c}\text { Tested } \\
\text { concentrations }\end{array}$ & Results & Authors \\
\hline HSYA* & $\begin{array}{l}\text { In vitro: tyrosinase, } \\
\text { l-DOPA }\end{array}$ & $\begin{array}{l}\text { Tyrosinase activity } \\
\text { assay }\end{array}$ & $\begin{array}{l}\text { Change in } \\
\text { absorbance } \\
\text { per min at } \\
475 \mathrm{~nm}\end{array}$ & $\begin{array}{l}0,0.5,1.5, \text { and } \\
2.5 \mathrm{mM} \text { HSYA }\end{array}$ & $\begin{array}{l}\text { Inhibition of l-DOPA oxidation, } \\
\text { reduction of tyrosinase activity }\end{array}$ & {$[43]$} \\
\hline \multirow{4}{*}{ HSYA } & \multirow{4}{*}{$\begin{array}{l}\text { In vitro: INS-1 (rat } \\
\text { insulinoma cells) }\end{array}$} & CAT kit & $\begin{array}{l}\text { CAT mRNA } \\
\text { level }\end{array}$ & \multirow{4}{*}{800 mM HSYA } & \multirow{4}{*}{$\begin{array}{c}\text { Increase of SOD, GSH-Px, and CAT } \\
\text { levels; decrease of ROS and MDA } \\
\text { levels }\end{array}$} & \multirow{4}{*}[44]{} \\
\hline & & GSH-Px kit & $\begin{array}{l}\text { GSH-Px } \\
\text { mRNA level }\end{array}$ & & & \\
\hline & & SOD kit & $\begin{array}{l}\text { SOD mRNA } \\
\text { level }\end{array}$ & & & \\
\hline & & MDA kit & MDA level & & & \\
\hline \multirow{5}{*}{ HSYA } & \multirow{5}{*}{ In vivo: mice } & $\begin{array}{l}\text { Lipid peroxidation } \\
\text { MDA assay kit }\end{array}$ & MDA level & \multirow{5}{*}{$\begin{array}{l}3.5 \mathrm{mg} \mathrm{kg}^{-1} \\
\text { HSYA in } 0.1 \mathrm{ml} \\
\quad \text { injection }\end{array}$} & $\begin{array}{l}\text { Control: approx. } 21 \mathrm{nmol} / \mathrm{mg} \text {; DHEA } \\
+ \text { HSYA = approx. } 22 \mathrm{nmol} / \mathrm{mg}\end{array}$ & \multirow{5}{*}[46]{} \\
\hline & & $\begin{array}{l}\text { GSH and GSSG } \\
\text { assay kit }\end{array}$ & $\begin{array}{l}\text { GSH/GSSG } \\
\quad \text { ratio }\end{array}$ & & $\begin{array}{c}\text { Control: approx. } 7.7 \text {; DHEA+ } \\
\text { HSYA = approx. } 6.4\end{array}$ & \\
\hline & & SOD assay kit & $\begin{array}{l}\text { SOD } \\
\text { activities }\end{array}$ & & $\begin{array}{c}\text { Control: approx. } 246 \mathrm{U} / \mathrm{mg} \text {; DHEA+ } \\
\text { HSYA = approx. } 200 \mathrm{U} / \mathrm{mg}\end{array}$ & \\
\hline & & GSH-Px assay kit & $\begin{array}{l}\text { Activities of } \\
\text { GSH-Px }\end{array}$ & & $\begin{array}{l}\text { Control: approx. } 600 \mathrm{mU} / \mathrm{mg} \text {; DHEA } \\
\text { + HSYA = approx. } 500 \mathrm{mU} / \mathrm{mg}\end{array}$ & \\
\hline & & CAT assay kit & $\begin{array}{l}\text { Activities of } \\
\text { CAT }\end{array}$ & & $\begin{array}{c}\text { Control: approx. } 59 \mathrm{U} / \mathrm{mg} \text {; DHEA+ } \\
\text { HSYA = approx. } 38 \mathrm{U} / \mathrm{mg}\end{array}$ & \\
\hline & & Commercial assay & SOD activity & 5 mg/kg HSYA; & Control = 199; HSYA = 125 & \\
\hline HSYA & In vivo: rats & $\begin{array}{l}\text { kits, fluorescence } \\
\text { spectrophotometry }\end{array}$ & $\begin{array}{l}\text { Content of } \\
\text { MDA }\end{array}$ & $\begin{array}{l}\text { intraperitoneally } \\
\text { injected }\end{array}$ & Control $=7 ;$ HSYA $=12$ & {$[47]$} \\
\hline \multirow{2}{*}{$\begin{array}{l}\text { HSYA, } \\
\text { carthamine }\end{array}$} & \multirow{2}{*}{$\begin{array}{c}\text { In vitro: HepG2 cells } \\
\text { and } 3 \mathrm{~T} 3-\mathrm{L} 1 \\
\text { adipocytes }\end{array}$} & MDA assay kit & MDA level & \multirow{2}{*}{$\begin{array}{l}200 \mathrm{mg} / \mathrm{kg} / \mathrm{d} \text { SY } \\
\text { or HSYA; } \\
\text { injection }\end{array}$} & \multirow{2}{*}{$\begin{array}{l}\text { Increase of SOD activity and MDA } \\
\text { level (in a high-fat diet group) }\end{array}$} & \multirow[b]{2}{*}[50]{} \\
\hline & & SOD assay kit & $\begin{array}{c}\text { SOD } \\
\text { activities }\end{array}$ & & & \\
\hline HSYC & $\begin{array}{l}\text { In vitro: } \mathrm{H} 9 \mathrm{c} 2 \text { (rat } \\
\text { pheochromocytoma } \\
\text { cells) }\end{array}$ & $\begin{array}{l}\text { Antioxidative } \\
\text { effects against } \\
\mathrm{H}_{2} \mathrm{O}_{2} \text {-induced } \\
\text { cytotoxicity } \\
\end{array}$ & Cell viability & $60 \mu \mathrm{g} / \mathrm{ml}$ HYSC & $\begin{array}{l}71 \% \text { of control; control: vitamin C } \\
(1.1 \mathrm{mg} / \mathrm{ml})\end{array}$ & {$[52]$} \\
\hline \multirow{2}{*}{$\begin{array}{l}\text { Methanol } \\
\text { extract of } \\
\text { safflower } \\
\text { flowers }\end{array}$} & \multirow[b]{2}{*}{ In vivo: rats } & FRAP & $\begin{array}{l}\text { Optical } \\
\text { density at } \\
700 \mathrm{~nm}\end{array}$ & $\begin{array}{c}20 \mathrm{mg} / \mathrm{ml} \text {, } \\
40 \mathrm{mg} / \mathrm{ml}, \text { and } \\
80 \mathrm{mg} / \mathrm{ml}\end{array}$ & $0.749 ; 1.155 ; 1.532$ (respectively) & \multirow[b]{2}{*}[49]{} \\
\hline & & $\mathrm{DPPH}$ & $\begin{array}{l}\text { Absorbance } \\
\text { at } 517 \mathrm{~nm} \text {; } \\
\mathrm{IC}_{50}\end{array}$ & $\begin{array}{c}0.1 \mathrm{ml} \text { solution of } \\
\text { different } \\
\text { concentrations of } \\
\text { extract } \\
\end{array}$ & $\mathrm{IC}_{50}=0.36$ & \\
\hline \multirow{2}{*}{$\begin{array}{l}\text { Safflower } \\
\text { flower } \\
\text { extract, } \\
\text { HSYA, SYA }\end{array}$} & \multirow{2}{*}{$\begin{array}{l}\text { In vitro: } \mathrm{HuDe} \\
\text { (human dermal } \\
\text { fibroblasts) }\end{array}$} & ORAC & $\begin{array}{l}\text { Fluorescence } \\
\text { emission } \\
\text { intensity at } \\
530 \mathrm{~nm}\end{array}$ & \multirow{2}{*}{$\begin{array}{l}\text { Water extract } \\
(5 \%), \text { HYSA, } \\
\text { SYA }\end{array}$} & $\begin{array}{l}\text { Total antioxidant activity } 130.2 \pm \\
12.3 \mathrm{mmol} \text { TE/100 g; Trolox index } \\
\begin{array}{r}\text { HSYA }=7.1 \pm 0.3 ; \text { Trolox index SYA } \\
=2.1 \pm 0.1\end{array}\end{array}$ & \multirow{2}{*}[48]{} \\
\hline & & $\mathrm{DPPH}$ & $\begin{array}{l}\text { Absorbance } \\
\text { at } 517 \mathrm{~nm} \text {; } \\
\mathrm{IC}_{50}\end{array}$ & & $\begin{array}{c}\mathrm{IC}_{50} \text { extract }=13.4 \pm 1.0 \\
(\mu \mathrm{g} \mathrm{GAE} / \mathrm{ml}) ; \mathrm{IC}_{50} \mathrm{HSYA}=7.3 \pm 1.2 \\
(\mu \mathrm{g} \mathrm{HSYA} / \mathrm{ml}) ; \mathrm{IC}_{50} \mathrm{SYA}=30.3 \pm 2.9 \\
(\mu \mathrm{g} \mathrm{SYA} / \mathrm{ml})\end{array}$ & \\
\hline
\end{tabular}

${ }^{*}$ Symbols: CAT: catalase; DHEA: dehydroepiandrosterone; GSH/GSSG ratio: reduced glutathione/oxidized glutathione; GSH-Px: glutathione peroxidase; HSYA: hydroxysafflor A; HYSB: hydroxysafflor B; HYSC: hydroxysafflor C; MDA: malondialdehyde; SOD: superoxide dismutase; SYA: safflor A.

improved their permeability [62]. The substance is also effective in the treatment of cerebrovascular injuries resulting from heat stress, as it inhibited apoptosis and autophagy of nerve stem cells and stimulated proliferation [65]. HSYA inhibited the development of Parkinson's disease symptoms by regulating the level of $\alpha$-synuclein, which reduced 
TABLE 3: Studies on the use of HSYA for medical purposes (from 2018 to 2020).

\begin{tabular}{|c|c|c|c|}
\hline Test organism & Type of test/parameter & Concentration/dose & Authors \\
\hline \multirow[t]{2}{*}{$\begin{array}{l}\text { In vitro: LAD2 (human mast } \\
\text { cells) and MPMCs (mouse } \\
\text { peritoneal mast cells) }\end{array}$} & $\begin{array}{l}\text { Intracellular } \mathrm{Ca}^{2+} \text { mobilization assay/imaging with } \\
\text { excitation at } 488 \mathrm{~nm}\end{array}$ & $\begin{array}{c}50 \mu \mathrm{M}, 100 \mu \mathrm{M}, \text { and } 200 \mu \mathrm{M} \text { HSYA } \\
(\mathrm{pH} 7.4)\end{array}$ & \multirow{6}{*}{ [57] } \\
\hline & $\begin{array}{l}\text { MTT assay (cell viability assay)/absorbance at } \\
490 \mathrm{~nm} \\
\beta \text {-Hexosaminidase release assay/absorbance at } \\
405 \mathrm{~nm}\end{array}$ & \multirow{3}{*}{$50 \mu \mathrm{M}, 100 \mu \mathrm{M}$, and $200 \mu \mathrm{M}$} & \\
\hline \multirow[t]{2}{*}{$\begin{array}{l}\text { In vitro: LAD2 (human mast } \\
\text { cells) }\end{array}$} & $\begin{array}{l}\text { ELISA kits (human chemokine array } \\
\text { kits)/measurements of cytokines (levels of TNF- } \alpha \text {, } \\
\text { interleukin- (IL-) } 8 \text {, and MCP-1) }\end{array}$ & & \\
\hline & $\begin{array}{l}\text { LC-ESI-MS/MS (liquid chromatography- } \\
\text { electrospray ionization-tandem mass } \\
\text { spectrometry)/histamine release assay }\end{array}$ & & \\
\hline $\begin{array}{l}\text { In vitro: protein extracted from } \\
\text { LAD2 cells }\end{array}$ & $\begin{array}{l}\text { Western blot analysis (ECL kit)/protein expression } \\
\text { (transillumination) }\end{array}$ & & \\
\hline In vivo: mice & $\begin{array}{l}\text { Hindpaw swelling and extravasation assay/optical } \\
\text { density at } 620 \mathrm{~nm}\end{array}$ & $\begin{array}{c}0,2.5 \mathrm{mg} / \mathrm{kg}, 5 \mathrm{mg} / \mathrm{kg}, 10 \mathrm{mg} / \mathrm{kg} \text { HSYA } \\
\text { in saline }\end{array}$ & \\
\hline In vivo: mice & $\begin{array}{c}\text { ELISA kits/assay the levels of testosterone, estradiol, } \\
\text { progesterone, luteinizing hormone, follicle- } \\
\text { stimulating hormone, anti-Müllerian hormone } \\
\text { (AMH) }\end{array}$ & $3.5 \mathrm{mg} \mathrm{kg}^{-1} \mathrm{HSYA}$ in $0.1 \mathrm{ml}$, injection & {$[46]$} \\
\hline \multirow{3}{*}{ In vitro: INS-1 (rat insulinoma cells) } & Cell viability assay/absorbance at $570 \mathrm{~nm}$ & $200 \mu \mathrm{M}, 400 \mu \mathrm{M}, 800 \mu \mathrm{M}$, and $1600 \mu \mathrm{M}$ & \multirow{3}{*}[44]{} \\
\hline & $\begin{array}{l}\text { Western blot analysis/protein expression } \\
\text { (transillumination, band densities) }\end{array}$ & \multirow{2}{*}{$800 \mu \mathrm{M}$} & \\
\hline & $\begin{array}{l}\text { Insulin ELISA kit/glucose stimulated insulin } \\
\text { secretion }\end{array}$ & & \\
\hline \multirow{6}{*}{ In vivo: rats } & Anthrone method/hepatic glycogen in the liver & \multirow{6}{*}{$120 \mathrm{mg} / \mathrm{kg}$, for 8 weeks } & \multirow{6}{*}[59]{} \\
\hline & WST-8 method/glycogen synthase & & \\
\hline & Fasting blood glucose/glucometer test & & \\
\hline & $\begin{array}{l}\text { Oral glucose tolerance test/glucometer test }(0,30 \text {, } \\
60 \text {, and } 120 \text { minutes after oral administration with } \\
\text { glucose solution) }\end{array}$ & & \\
\hline & $\begin{array}{c}\text { Assay kits/fasting blood insulin, triglycerides, total } \\
\text { serum cholesterol, high-density lipoprotein } \\
\text { cholesterol, low-density lipoprotein cholesterol in } \\
\text { serum }\end{array}$ & & \\
\hline & Western blot analysis/protein expression & & \\
\hline $\begin{array}{l}\text { In vitro: } \mathrm{HK}-2 \text { (renal tubular } \\
\text { epithelial cells) }\end{array}$ & $\begin{array}{l}\text { Western blot analysis/protein expression } \\
\text { Cellular immunofluorescence assay/photographing } \\
\text { (fluorescent inverted microscope) }\end{array}$ & \multirow{4}{*}{$\begin{array}{l}\text { Doses of } 10 \mathrm{mg} / \mathrm{kg} \text { daily, intragastrically, } \\
\text { for } 6 \text { weeks }\end{array}$} & \multirow{4}{*}[60]{} \\
\hline \multirow{3}{*}{ In vivo: rats } & $\begin{array}{l}\text { ELISA kit/interleukin-6 levels, tumor necrosis } \\
\text { factor- } \alpha \text { level }\end{array}$ & & \\
\hline & $\begin{array}{l}\text { RT-qPCR assay (PCR)/relative expression level of } \\
\text { miRNA-140-5p }\end{array}$ & & \\
\hline & $\begin{array}{l}\text { BCA method (based on T-AOC and MDA } \\
\text { kit)/protein concentrations }\end{array}$ & & \\
\hline \multirow{3}{*}{$\begin{array}{l}\text { In vitro: } \mathrm{PC} 12 \\
\text { (pheochromocytoma cell line) }\end{array}$} & MTT assay/cell viability & $5 \mu \mathrm{M}, 10 \mu \mathrm{M}, 20 \mu \mathrm{M}, 40 \mu \mathrm{M}$, and $80 \mu \mathrm{M}$ & \multirow{4}{*}[63]{} \\
\hline & $\begin{array}{l}\text { Total mRNA (amount and purity)/absorbance at } \\
\qquad 260 / 280 \mathrm{~nm}\end{array}$ & \multirow[t]{2}{*}{$10 \mu \mathrm{M}$} & \\
\hline & Western blot/protein expression & & \\
\hline $\begin{array}{l}\text { In vitro: b.End3 (mouse brain } \\
\text { microvascular endothelial cells) }\end{array}$ & $\begin{array}{l}\text { Immunofluorescence assay/immunofluorescence } \\
\text { (confocal microscopy) }\end{array}$ & $20 \mu \mathrm{M}$ & \\
\hline
\end{tabular}


TABLe 3: Continued.

\begin{tabular}{lcc}
\hline Test organism & Type of test/parameter & Concentration/dose \\
\hline In vivo: rats & $\begin{array}{c}\text { Therapeutic effect in vivo/infarct area, ratio of } \\
\text { infarct volume to whole brain tissue }\end{array}$ & $10 \mathrm{mg} / \mathrm{kg}, 20 \mathrm{mg} / \mathrm{kg}$ \\
\hline & CCK-8 assay/absorbance at $450 \mathrm{~nm}$ & Authors \\
$\begin{array}{l}\text { In vitro: NSCs (neural stem cells } \\
\text { of rats) }\end{array}$ & $\begin{array}{c}\text { EdU assay/cell proliferation rate } \\
\text { Cell apoptosis/BD FACSCalibur flow cytometry } \\
\text { Western blot analysis/protein expression }\end{array}$ & $1 \mu \mathrm{M}, 5 \mu \mathrm{M}$, and 10 $\mu \mathrm{M}$ \\
\hline In vivo: mice & Hanging wire test/hanging time & {$[65]$} \\
\hline
\end{tabular}

IPGTT (intraperitoneal glucose tolerance test), IPITT (intraperitoneal insulin tolerance In vivo: mice test)/glucose and insulin tolerance tests

2-DDCt method/expression of antioxidant enzymes in the liver and adipose tissue

2-DDCt method/expression of antioxidant enzymes

ALP activity assay/percentage of ALP activity

In vitro: HepG2 cells and 3T3-L1 adipocytes
Caspase colorimetric assay kit/caspase-3 activity assay

Annexin V-FITC/IP staining kit/percentage of apoptotic cells

Western blot analysis/protein expression

$\mathrm{HOP}^{*}$ and $\mathrm{HOM}^{*}$ concentration in serum

ELISA kits/concentrations of inflammatory cytokines (IL-6, IL-1 $\beta$, and TNF- $\alpha$ )

In vitro: HSAEC1-KT HSAECs (human small airway epithelial cells)
Western blot analysis/protein expression

Calcium-sensitive fluorescent probe Fluo-

3/AM/intracellular calcium ion concentration

Dual-luciferase reporter assay/transcriptional activities of NF- $\kappa$ B and AP1
$200 \mathrm{mg} / \mathrm{kg} / \mathrm{d}$ HSYA, for 10 weeks

3T3-L1 adipocytes: 10, 50, and $100 \mathrm{mg} / \mathrm{l}$ HSYA for $24 \mathrm{~h}$; HepG 2 cells: 10, 50, and $100 \mathrm{mg} / \mathrm{l} \mathrm{SY}{ }^{*}$ for $24 \mathrm{~h}$

Double-staining method (with Tcc and Evans blue stains)/infarct size

In vivo: rats

\section{ELISA kits/cTnI, IL-6, and LdH levels}

Flow cytometry analysis, TUNEL (terminal deoxynucleotidyl transferase-mediated dUTP nickend labeling)/apoptosis level

ELISA/caspase- 3 activity

CCK-8/cell viability

\section{$5 \mathrm{mg} / \mathrm{kg}$ HSYA; intraperitoneally} injected

Western blot analysis/protein expression

Span diagnostic reagent kit/serum glutamic oxaloacetic transaminase

$20 \mu \mathrm{M}$ cells)

Span diagnostic reagent kit/serum alkaline phosphatase

Agappe diagnostic kit/serum total bilirubin

In vivo: mice

In vitro: $\mathrm{H} 22$ (murine hepatoma cells), HepG2 (human hepatocellular carcinoma cells)
SP kit (MMP-2, MMP-9, and COX-

2)/immunohistochemical analysis

Growth inhibition assay-CCK-8/rate of growth inhibition ( $\mathrm{IC}_{50} 80 \mu \mathrm{M}$ for $72 \mathrm{~h}$ )

Western blot analysis/protein expression

Clonogenic assay/optical density at $570 \mathrm{~nm}$
$1.125 \mathrm{mg} / \mathrm{kg}, 2.25 \mathrm{mg} / \mathrm{kg}$, intraperitoneally, for 14 days

$40,60,80,120$, and $160 \mu \mathrm{M}$, for 24,48 , 
TABLe 3: Continued.

\begin{tabular}{|c|c|c|c|}
\hline Test organism & Type of test/parameter & Concentration/dose & Authors \\
\hline & $\begin{array}{l}\text { Wound healing assay/cell migration, via Image-Pro } \\
\text { Plus }\end{array}$ & & \\
\hline \multirow{5}{*}{$\begin{array}{l}\text { In vitro: KYSE- } 30 \text { cells } \\
\text { (esophageal cancer cells) }\end{array}$} & $\begin{array}{l}\text { CCK-8 assay/proliferative activity (optical density } \\
\text { of cells) }\end{array}$ & $\begin{array}{c}0,1,1,10,20 \text {, and } 50 \mu \mathrm{M} \text {, for } 24,48 \text {, and } \\
72 \mathrm{~h}\end{array}$ & \multirow{5}{*}{ [92] } \\
\hline & $\begin{array}{l}\text { Microscopic measurement of the number of } \\
\text { cells/cell invasion and cell migration assays }\end{array}$ & \multirow{4}{*}{$20 \mu \mathrm{M}$, for $24 \mathrm{~h}$} & \\
\hline & $\begin{array}{l}\text { Flow cytometric analysis of cell apoptosis/apoptotic } \\
\text { rate of cells }\end{array}$ & & \\
\hline & Western blot analysis/protein expression & & \\
\hline & $\begin{array}{c}\text { Serum levels of AFP, ALT, TBIL, and } \\
\text { ALB/automatic analyzer }\end{array}$ & & \\
\hline \multirow{3}{*}{$\begin{array}{l}\text { In vitro: } \mathrm{A} 2780 / \mathrm{DDP} \text { (ovarian } \\
\text { carcinoma cells) }\end{array}$} & Cytotoxicity assay (CCK-8)/cell survival rates & & \multirow{5}{*}[45]{} \\
\hline & $\begin{array}{l}\text { RTCA test (real-time cellular analysis)/monitoring } \\
\text { cell proliferation }\end{array}$ & $1 \mathrm{mg} / \mathrm{ml}$ & \\
\hline & $\begin{array}{c}\text { Flow cytometry (Guava EasyCyte Plus)/evaluation } \\
\text { of apoptosis }\end{array}$ & $1 \mathrm{mg} / \mathrm{ml}$, for 24 & \\
\hline \multirow[t]{2}{*}{ In vivo: mice } & $\begin{array}{l}\text { Electronic digital caliper/weekly measurement of } \\
\text { tumor size and volume }\end{array}$ & \multirow[t]{2}{*}{$\begin{array}{l}1.1 \mathrm{~g} / \mathrm{kg} \text { body weight; once every three } \\
\text { days, for five weeks }\end{array}$} & \\
\hline & Western blot analysis/protein expression & & \\
\hline \multirow{3}{*}{$\begin{array}{l}\text { In vitro: SKOV-3 (ovarian } \\
\text { carcinoma cells) }\end{array}$} & Cell proliferation assay kit/cell proliferation & $10,20,50,100$, and $150 \mathrm{mg} / \mathrm{l}$, for $72 \mathrm{~h}$ & \multirow{3}{*}[90]{} \\
\hline & CellTiter-Blue Cell Viability kit/cell viability assay & $10,20,50,100$, and $150 \mathrm{mg} / \mathrm{l}$, for $48 \mathrm{~h}$ & \\
\hline & $\begin{array}{l}\text { Western blot analysis (protein concentration assay } \\
\text { kit)/protein expression }\end{array}$ & $10,20,50,100$, and $150 \mathrm{mg} / \mathrm{l}$ & \\
\hline
\end{tabular}

n.d.: no date; SY: carthamine; HOP: hydroxyproline; HOM: hexosamine; CCK-8: cell counting kit.

synthesis of dopamine and retarded intracellular degradation of brain cells [66].

4.6. Skin Discolorations. HSYA has proven effective in the treatment of skin hyperpigmentation and hypopigmentation. The substance formed complexes with tyrosinase altering its activity, and as an end result, it inhibited production of melanin from tyrosine. This resulted in homogenization of the skin color [43].

4.7. Overweight and Obesity. HSYA reduces obesity in mice and rats. When administered orally, it resulted in a change of composition of the diet-dependent intestinal microflora. As a result of this, certain bacteria groups had markedly increased counts, while other reduced, which affected the course of digestive processes and enhanced the function of the digestive tract (i.e., the intestines), as well as systemic metabolism. The level of lysophosphatidylcholines (lyso PCs), L-carnitine, and sphingomyelin increased, whereas that of phosphatidylcholines decreased. This resulted in reduced amount of fat accumulated, restored glucose homeostasis, alleviation of insulin resistance, and reduced amount of inflammations in the organism [67]. What is more, HSYA stimulated the production of antioxidative enzymes present in the liver and adipose tissue, which reduced obesity in mice, while intercurrent administration of HSYA and safflower yellow (syn.: carthamine yellow) increased the level of mRNA of antioxidative enzymes and resulted in increased activity of superoxide dismutase (SOD) in the liver [50]. HSYA increased synthesis of hormone-sensitive lipase (HSL) and affected its activity, which inhibited adipocyte proliferation [68].

4.8. Skeletal System. During in vitro studies, safflower yellow (SY) pigment increased migration of endothelial cells of the umbilical vein and produced increase in angiogenesis and differential of bone cells via increasing levels of HIF-1 $\alpha$, VEGF, Ang-2, ALP, Runx2, and OPN-1, directly affecting the $\mathrm{pVHL} / \mathrm{HIF}-1 \alpha / \mathrm{VEGF}$ signaling pathway, which enables easier treatment of bone fractures [69]. Favorable impact of water-soluble polysaccharides isolated from safflower on the cells of the head of the thigh bone in rats and mice treated with steroid drugs (prolonged use of steroids results in i.a. thigh bone head osteonecrosis) was demonstrated [69-72]. Polysaccharides inhibited the activation of caspase- 3 participating in the processes of cell apoptosis, which resulted in increased viability of thigh bone head cells and contained osteoblast apoptosis [70]. Following a 60-day treatment with a polysaccharide of beta-d-glucan group with $(1 \rightarrow 3)$ bonds, regression of histopathological changes, reduced number of cells involved in apoptosis, and adipose cells in the bone marrow were observed, which was accompanied by reduced expression of Bax protein and caspase-3, with concomitant increase in Bcl-2 protein expression [72]. Similar effect was produced by water-soluble polysaccharide containing repeating $1,4,6-\beta$-Glcp skeleton bonded with T-B-Glpc in C6 
TABLE 4: Studies on the use of the substances contained in safflower flowers, except HSYA, for medical purposes (from 2018 to 2020).

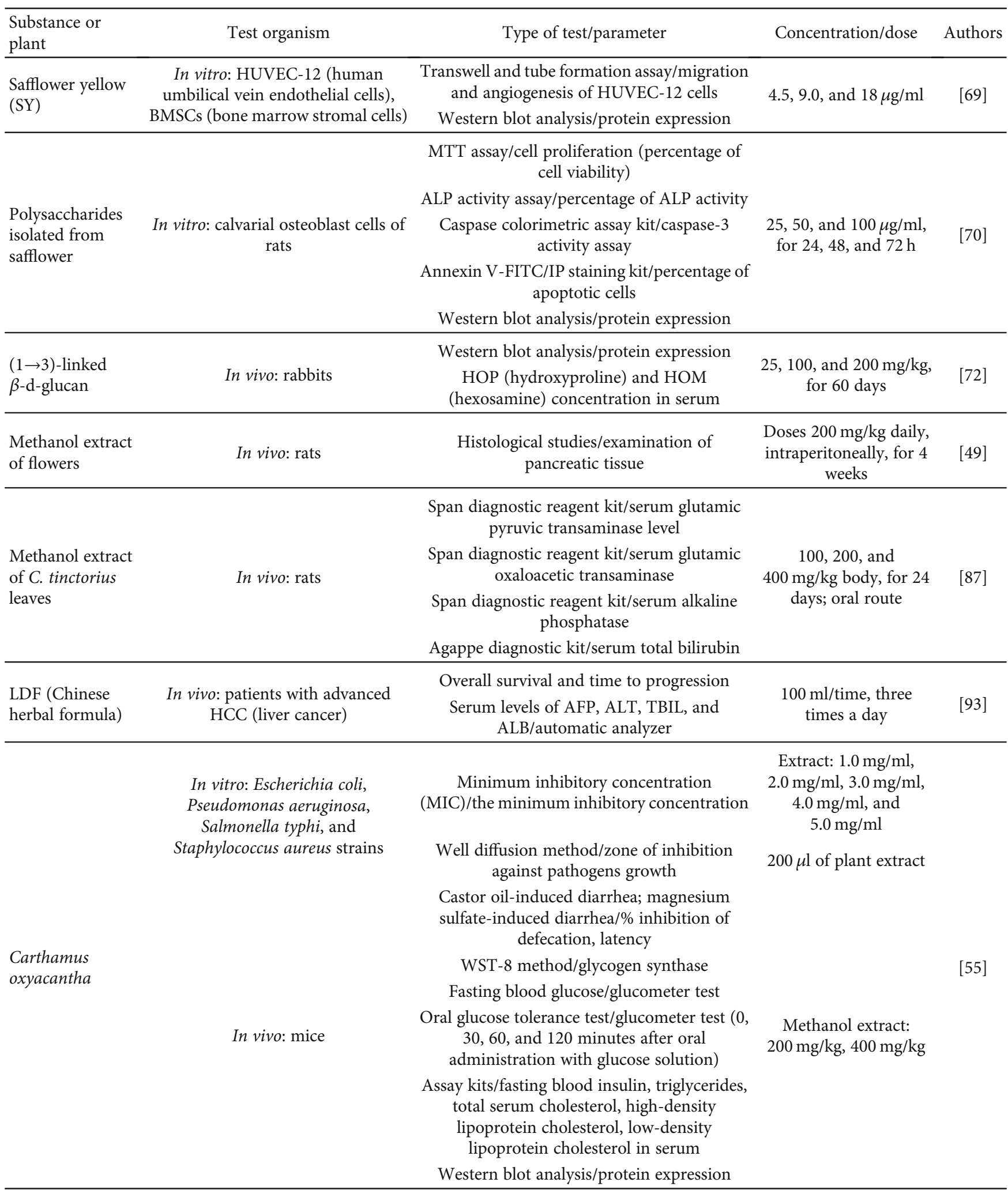

position. It enhanced bone mineral density and reduced the amount of histopathological changes, the amount of void gaps in the bone, and the apoptosis indicator for the osteo- cytes of the thigh bone head. It further resulted in increased level of hydroxyproline in blood serum and drop in the hexosamine blood level [71]. 
TABle 5: Action mechanisms by safflower substances.

\begin{tabular}{|c|c|c|c|}
\hline Activity & Result of mechanism & Mechanism & Authors \\
\hline $\begin{array}{l}\text { Prevention of } \\
\text { anaphylaxis }\end{array}$ & $\begin{array}{l}\text { (i) Inhibition of mast cell degranulation } \\
\text { (ii) Reduction the activation of the PLC } \gamma \text {-PKC-IP3 } \\
\text { signaling pathway }\end{array}$ & $\begin{array}{l}\text { (i) Inhibition of } \mathrm{Ca}^{2+} \text { flow } \\
\text { (ii) Inhibition of MCP-1, IL- } 8, \beta \text {-hexosaminidase, } \\
\mathrm{HA} \text {, and TNF- } \alpha \text { release } \\
\text { (iii) Inhibition of phosphorylation of PLC } \gamma 1 \text {, IP3R, } \\
\text { PKC, Akt, P38, and Erk1/2 }\end{array}$ & {$[57]$} \\
\hline
\end{tabular}

(i) Reversion of the expression of genes Star, Hsd3b1, Cyp11a1 (increase), and Cyp19a1 (reduction)
Alleviation of polycystic ovary syndrome
(i) Reduction of cysts
(ii) Regulation of the hormonal balance
(iii) Restoration of the ovulation cycle

(ii) Increase in antioxidant enzyme activities (SOD, GSH-Px, and CAT)

(iii) Regulation of the level of T, E2, FSH, P4, and $\mathrm{AMH}$ and the ratio of $\mathrm{LH} / \mathrm{FSH}$ in serum

(iv) Reduction of MDA level and enhanced GSH content and GSH/GSSG ratio

(i) Induction of cisplatin sensitivity by JNK and P38 MAPK signaling pathway

(i) Inhibition of cancer cell proliferation

(i) Inhibition of cancer cell proliferation

(ii) Induction of cancer cell apoptosis (i) Inhibition of tumor angiogenesis

(i) Induction of autophagy in cancer cells by regulating Beclin 1 and ERK expression

(i) Induction of apoptosis of tumor cells by regulating the NF- $\kappa \mathrm{B}$ signaling pathway (inhibition of the tumor growth)

(i) Inhibition of the activation of the pyroptotic pathway and apoptosis of injured nerves

(ii) Activation of damage mitigating factor

Alleviation of damage and brain injuries (i) Reduction of the apoptosis and autophagy of neural stem cells by modulation of the p38/MAPK/MK2/Hsp27-78 signaling pathway

(ii) Stimulation of the cell proliferation

(i) Inhibition of dopamine synthesis

(ii) Promotion $\alpha$-syn clearance by regulating autophagy (i) Increase in P-JNK and P-38 levels

(i) Inhibition of Skov3 cell proliferation

(ii) Reduction of WSB1 expression

(iii) Inhibition of Erk1/2 expression and Erk phosphorylation

(i) Inhibition of the MCF-7 cell cycle at the S phase

(ii) Reduction of CDK2, cyclin D1, and cyclin E levels

(iii) Reduction of p-PI3K, PI3K, AKT, and p-AKT levels

(i) Inhibition of p38 MAPK phosphorylation

(ii) Reduction of MMP-2 and MMP-9 levels

(iii) Reduction of COX-2 expression by p38MAPK/ATF-2 signaling pathway (by inhibition of p38MAPK phosphorylation)

(iv) Increase of the caspase- 3 cleavage in tumor cells

(i) Increase in Beclin 1 and LC3-II expression in tumor cells

(ii) Reduction of phosphorylated ERK1/2 expression and p62 level in tumor cells

(i) Inhibition of the expression of ICAM1, MMP9, TNF- $\alpha$, and VCAM1

(ii) Increase in the expression of $\mathrm{p}-\mathrm{I} \kappa \mathrm{B} \alpha$ and $\mathrm{pP} 65$

(i) Reduction of cytokine expression (NLRP3, ASC, caspase-1, GSDMD, IL- $1 \beta$, IL- 18 , LDH, NF- $\kappa$ B, and p-p56)

(ii) Changes in activation of the NF- $\kappa \mathrm{B}$ signaling pathway

(i) Reduction of p38 and Hsp27-78 phosphorylation and MK-2, Bax, cleaved caspase-3, LC3-II, and mTOR phosphorylation expression

(ii) Increase in $\mathrm{Bcl}-2$ and $\mathrm{p} 62$ expression

(i) Increase in the formation of autophagosomes

(ii) Increase of TH, p-JNK1/JNK1, Beclin 1, Atg7, Atg12-5, and p-Bcl-2/Bcl-2 expression and the LC3-II/LC3-I ratio

(iii) Reduction of $\alpha$-syn expression 
TABLe 5: Continued.

\begin{tabular}{ll}
\hline Activity & Result of mechanism \\
\hline & $\begin{array}{l}\text { (i) Inhibition of JNK/c-Jun signaling pathway } \\
\text { (ii) Alleviation of oxidative damage }\end{array}$ \\
$\begin{array}{l}\text { Alleviation of } \\
\text { diabetes } \\
\text { complications }\end{array}$ & $\begin{array}{l}\text { (i) Promotion of PI3K and Akt activation } \\
\text { (ii) Inhibition of the apoptosis of pancreatic } \beta \text {-cells }\end{array}$ \\
& $\begin{array}{l}\text { (i) Reduction of renal fibrosis } \\
\text { (ii) Regulation of the TLR4/NF- } \kappa \mathrm{B}(\mathrm{p} 65) \text { pathway } \\
\text { and miRNA-140-5p level }\end{array}$ \\
\hline
\end{tabular}

Mechanism

Authors

(i) Inhibition of p-JNK and p-c-Jun activation

(ii) Reduction of phosphorylation of JNK and c-Jun

(iii) Reduction of cleaved parp and cleaved caspase-3 levels

(i) Increase of PI3K, AKT, and p-AKT expressions

(ii) Increase in contents of hepatic glycogen and glycogen synthase in the liver

(i) Increase of miRNA-140-5p mRNA, BG, $24 \mathrm{~h} \mathrm{UP}$, TC, TG, T-AOC, MDA, IL-6, TNF- $\alpha$, TLR4, NF- $\kappa$ B(p65), NLRP3, Notch2, and Col-IV

(i) Increase of CAT, GSH-Px, MDA, and SOD activities

Protection of the

(i) Protection of the liver and other organs against aging

(ii) Reduction of the mRNA, protein level of cyclindependent kinase inhibitor p16 and

digestive system phosphorylation of $\mathrm{pRb}$

(iii) Increase in CDK4/6 protein expression

(i) Protection of the liver against damage

(i) Reduction of ALT, ALP, AST, and total bilirubin levels

(i) Change in platelet activation pathway

(i) Regulation of core genes: PRKACA, PIK3R1, MAPK1, PPP1CC, PIK3CA, and SYK

(i) Inhibition of caspase-3 activity (reduction of H/Rinduced apoptosis)

(ii) Reduction of Janus kinase 2 (JAK2)/signal transducer and activator of transcription 1 (STAT1) activity

Protection and treatment of cardiovascular diseases (i) Inhibition of activation of the JAK2/STAT1
pathway

(iii) Reduction of releases of cTnI, IL-6, and LdH

(iv) Change of expression levels of Bcl-2-associated X protein, Bcl-2, cleaved caspase-3, Fas ligand, and tumor necrosis factor receptor superfamily member 6 (Fas)

(i) Inhibition of the PKA and NO production

(i) Effect on vasodilation

(ii) Activation of $\mathrm{p}$-eNOS expression

(iii) Change of the influx of $\mathrm{Ca}^{2+}$ (TRPV4-dependent)

(i) Increase of ALP, Ang-2 (Angiopoietin-2), HIF-1 $\alpha$ (hypoxia inducible factor- $1 \alpha$ ), OPN-1 (osteopontin-1), Runx2 (runt-related transcription factor 2), and VEGF (vascular endothelial growth factor) levels

Protection of

(i) Regulation of pVHL/HIF-1 $\alpha /$ VEGF pathway

(ii) Increase in angiogenesis and bone cell differentiation

skeletal system

(iii) Inhibition of HIF- $1 \alpha$ expression

(i) Increase in osteoblast differentiation

(ii) Inhibition of osteoblast apoptosis

(ii) Inhibition of SY-induced proliferation, migration, and angiogenesis

(i) Inhibition of caspase- 3 activity (change in caspase3-dependent signaling pathway)

(i) Changes in the expression of interleukin- (IL-) $1 \beta$ and IL-6, inflammatory signaling pathways, monolayer permeability of HSAECs, and tumor necrosis factor alpha

(ii) Reduction of inflammatory factor expression and nuclear factor- $\kappa \mathrm{B}$ activation

(iii) Inhibition of activator protein-1, protein kinase $C$, and mitogen-activated protein kinase expression 
TABLe 5: Continued.

\begin{tabular}{|c|c|c|c|}
\hline Activity & Result of mechanism & Mechanism & Authors \\
\hline \multirow{2}{*}{$\begin{array}{l}\text { Reduction of } \\
\text { overweight and } \\
\text { obesity }\end{array}$} & $\begin{array}{l}\text { (i) Change in the composition of intestinal } \\
\text { microflora } \\
\text { (ii) Restoration of glucose homeostasis } \\
\text { (iii) Alleviate insulin resistance }\end{array}$ & $\begin{array}{l}\text { (i) Changes in pathways of sphingolipid and } \\
\text { glycerophospholipid metabolisms } \\
\text { (ii) Increase of L-carnitine, lysophosphatidylcholine, } \\
\text { and sphingomyelin levels } \\
\text { (iii) Reduction of phosphatidylcholines }\end{array}$ & {$[67]$} \\
\hline & $\begin{array}{l}\text { (i) Increase in the synthesis of antioxidant enzymes } \\
\text { in adipose tissue and in the liver }\end{array}$ & $\begin{array}{l}\text { (i) Increase of expression of antioxidant enzymes and } \\
\text { Nrf2 in adipocytes, liver tissue, and HepG2 cells } \\
\text { (ii) Regulation of glucose metabolism and liver } \\
\text { function }\end{array}$ & {$[50]$} \\
\hline
\end{tabular}

4.9. Respiratory System. HSYA produced reduction of vessel permeability, reduction of the amount of blood platelets in plasma, and reduced their aggregation in the lungs of rats with prolonged exposure to car exhaust fumes, which considerably mitigated lung injury and reduced the likelihood of other lung diseases [73]. HSYA had protective effect on the respiratory system of guinea pigs, and it alleviated the symptoms of acute lung injury, chronic obstructive pulmonary disorder, and asthma caused by ovalbumin. It inhibited the action of platelet-activation factor (PAF) in the airway epithelium and mitigated inflammatory processes by blocking synthesis of triggers: protein- 1 activator, nuclear $\kappa \mathrm{B}$ activation factor, protein kinase $\mathrm{C}$, and protein kinases activated by mitogen [74].

4.10. Circulatory System. In China, HSYA is recommended for the treatment of angina pectoris [75] and other circulatory disorders [76]. This substance reduced myocardial damage following ischemic/reperfusion injury occurring in infarction $[47,77-79]$. It has the capability to inhibit caspase-3 activity, reducing $\mathrm{H} / \mathrm{R}$ induced apoptosis and enhancing cell resistance to oxidative stress [47]. It suppressed TLR4 signaling (by blocking the pathway including Toll-like receptors) and lowered inflammatory cytokine secretion, which inhibited development of inflammation within the damaged cardiac muscle [77]. The protective effect of HSYA consisted in its inclusion in the course of the PI3K/Akt/hexokinase II signaling pathway to activate hexokinase II proteins, which consequently reduced cell apoptosis [79]. Intravenous administration of carthamine yellow (CY) also has favorable impact on the treatment of the effects of acute myocardial infarction. It supported angiogenesis, which increased the number of unobstructed capillaries in the area adjacent to the infarction site and favored revascularization and restoration of normal function of the cardiac muscle [80]. Moreover, research confirmed positive effect of hydroxysafflor C (HSYC) on the prevention of cardiovascular diseases [52].

Substances isolated from safflower flowers have therapeutic effect on blood pressure problems. HSYA reduced systolic pressure in the left ventricle in rats with pulmonary hypertension [78]. This pigment produced dilation of vessels in the mesenteric artery in rats which resulted in reduced blood pressure thanks to elevated transport of $\mathrm{Ca}^{2+}$ ions to endothelial cells, eNOS phosphorylation, and nitric oxide synthesis [81]. The strongly dilating effect of HSYA on vessels is further related to its activation of the KV channel in the pulmonary vascular smooth muscle cells [82]. Additionally, positive impact of carthamine yellow (CY) on the blood coagulation process was revealed $[62,83]$. CY reduced blood viscosity, plasma viscosity, and erythrocyte aggregation index, which resulted in reduction in blood pressure. Decrease in hematocrit and platelet aggregation was observed with increased CY dose. This may pose a chance to prevent embolisms by increasing blood liquidity. The study authors pointed to the fact that using CY as a pigment in foods may pose risk in the case of persons with hemorrhages, but they estimated it to be minor [83].

4.11. Digestive System. HSYA inhibited liver cell fibrosis [84, 85] resulting from induction of apoptosis of stellate cell responsible for the development of disease by blocking activation of expression of the genes regulated by ERK1/2 (including Bcl-2, cytochrome C, caspase-9, and caspase-3 [84]) and thanks to PPAR activation, increasing activity of antioxidative enzymes, increasing expression of PPAR and MMP-2, reducing expression of TGF-1 and TIMP-1, and lowering $\alpha$-SMA level [85]. In the organisms of aging mice and mice exposed to pathologic changes, HSYA fulfilled protective role for the liver and other organs, reducing the level of mRNA and the amount of cyclin-dependent protein kinase inhibitor p16 [86]. Similarly, the extract obtained from safflower leaves can fulfill protective role towards liver exposed to injury due to administration of antituberculous drugs (this pigment produced substantial reduction of AST, ALT, and ALP parameters and total bilirubin). Studies on methanol extract further revealed presence of lupeol (a triterpenoid of anti-inflammatory and antineoplastic significance) and $\beta$-sitosterol (phytosterol producing, among others, poorer absorption of cholesterol in the digestive tract [87]). A herbal blend including dried safflower flowers and Salvia miltiorrhiza root administered as an injection (Danhong injection) alleviated gastric mucosal lesions caused by administration of salicylic acid, even with prolonged exposure to the drug. It reduced pepsin production and reduced the level of reactive oxygen species in the gastric mucosa [88].

4.12. Neoplastic Diseases. HSYA inhibits the development of cancers [45, 51, 89-93]. It inhibited angiogenesis of liver cancer by blocking the ERK/MAPK $[89,94]$ and $\mathrm{NF} /{ }_{\mathrm{K}} \mathrm{B}$ HSYA signaling pathway in mice [89] and via inhibiting p38MAPK phosphorylation [91]. Administration of HSYA enhanced spleen and thymus indicators and immune system function 
[89] and resulted in reduced viability, proliferation, and migration of HepG2 tumor cells [91]. By affecting the NF- $\kappa$ B signaling pathway, HSYA also inhibited the development of malignant esophageal cancer cells: their proliferation and migration were blocked and accelerated apoptosis was observed (blockage of ICAM1, MMP9, TNF- $\alpha$, and VCAM1 expression and stimulation of phosphor-nuclear transcription factor kappa B p65 expression) [92]. Similarly, positive effect was observed following application of a herbal mixture containing, among others, Carthamus tinctorius flowers on the level of HFP, ALT, and TBIL hepatic indicators. This multicomponent product inhibited development of hepatocellular carcinoma (HCC) in humans and increased overall survival, as well as minimized the risk of complications [93].

HSYA reversed drug resistance to chemotherapy drugs of ovarian carcinoma cells in mice $[45,90]$ by unblocking MAPK signaling pathways in resistant cells [45]. Thanks to the expression of WSB1 gene, the tumor cell proliferation was also inhibited [90]. Furthermore, the pigment exhibited anti-inflammatory and antioxidative action, affected survival rate of cells, and inhibited tumor angiogenesis [45].

HSYB, an isomer of HSYA, inhibited proliferation of breast cancer cells (MCF-7) in humans and reduced survival rate and proliferation of tumor cells by blocking their cellular cycle in S phase. Increased apoptosis of tumor cells was linked to reduced level of cyclin D1, cyclin E, CDK2, p$\mathrm{PI} 3 \mathrm{~K}, \mathrm{PI} \mathrm{K}, \mathrm{AKT}$, and p-AKA proteins in MCF-7 cells and reduced level of Bcl-2 [51].

4.13. Adverse Symptoms. Doses up to $2000 \mathrm{mg} / \mathrm{kg}$ body weight of carthamus red are safe (no toxicity was found [95]); however, with long-term oral administration of higher concentrations of safflower flower extracts $(200 \mathrm{mg} / \mathrm{kg}$ for 35 days), changes in the functioning of the male reproductive system [96, 97], pharyngitis, and nosebleeds [98] were observed. Injection of safflower extract induced allergy reactions $[99,100]$, and with intraperitoneal administration of HSYA, slight changes in the functioning of the kidneys (at a dose of $180 \mathrm{mg} / \mathrm{kg}$ [101]) and liver [102] were noted.

\section{Applicability of Safflower Bioactive Substances in Food Industry}

Importance of $C$. tinctorius is primarily linked to the commercial use of seed and flower petals. Seeds are mainly used for the production of edible oil and for feed purposes, whereas flower petals are used to obtain dyes applied in apparel, food and cosmetic industry, medicine, and in painting $([32,103]$, Henry and Francis 1996 by [104], [105, 106]), while aboveground parts of plants are used to produce animal feed [107].

Due to the high content of carotene, riboflavin, and vitamin $C$ in the green parts, in India this plant is cultivated as leaf vegetable [108]. Safflower leaves are characterized by perceptible bitter taste [54]. In order to retain the valuable leaf components, they should not be subject to prolonged treatment in high temperature, suggesting that they can (after a possible brief blanching) constitute an interesting addition to fresh salads, dips, and cold soups, adding a bitter flavor (Figure 2).
Safflower flowers also carry a potentially high significance for food production, because they can constitute an ingredient enriching meals with nutrients. Flower petals contain all necessary amino acids, except tryptophan. Flowers of thornless cultivars are popular already: they have been shown to be rich in protein, sugars, calcium, iron, magnesium, and potassium. By using these properties, teas, whose main ingredient are $C$. tinctorius petals, were composed and popularized in China and India (Singh (2005a) by [10]). However, these petals can comprise an interesting ingredient of fresh salads, which does not only enrich the sensory values of products, including flavors (associated with volatile oils present in the flowers) or values linked to the bright coloration of the petals. Such petals could also constitute an additional source of valuable bioactive dietary nutrients (Figure 2). Safflower petals were used on a mass scale to obtain pigments for food products, but when less expensive synthetic pigments were made popular, the use of natural colorants was markedly lowered. This approach changed with the introduction of legal regulations on the substances authorized for use in food production in many countries, when the interest in natural sources of colorants increased again [10].

Thus far, mainly carthamine and carthamidin have been used in food production (Figure 2). Carthamine, due to its poor water solubility, was used to dye chocolate, while carthamidin can be found in colorful juices, jellies, and candies (after [103]). Carthamine is unstable in aqueous solutions and decomposes very easily at elevated temperature and in alkaline solutions (Fatahi et al. (2009) by [109]); therefore, a method for stabilizing this pigment was developed using natural ionic liquids and deep eutectic solvents, which are natural primary metabolites [109]. These are usually sugars and sugar alcohols, amino acids and amines, and organic acids, which possess several hydroxyl, carboxyl, or amine groups [110]. Such solutions protect carthamine against the negative impact of light, temperature, and prolonged storage [109]. The use of natural dyes obtained from safflower flowers (yellow safflower extract) in food production is allowed in the EU and Asia, but banned in the US [111], although producers of safflower yellow recommend them for use in meat preparations, cake coatings and desserts, jellies, candies, and canned vegetables and fruit as well as flour and rice products and carbonated drinks [112].

The applicability of carthamidin as an ice cream dye was investigated. Addition of this pigment had positive effect on sensory evaluation and chemical composition of products. The highest sensory acceptability is characterized by ice cream containing $0.06 \mathrm{ml}$ of carthamidin; increasing and decreasing the content of this substance deteriorated results of sensory evaluation, particularly for flavor, color, and texture of product. Addition of the pigment caused a slight increase in the moisture and the content of protein, lipids, carbohydrates, and ash. The authors of this study pointed to the health benefits of using this natural pigment [30]. The production technology of Pedha was also developed and tested with the addition of carthamine as the pigment that gives the yellow color of this traditional Indian sweet snack [113]. The addition of carthamine resulted in a minor increase of moisture and protein and ash content and minor 


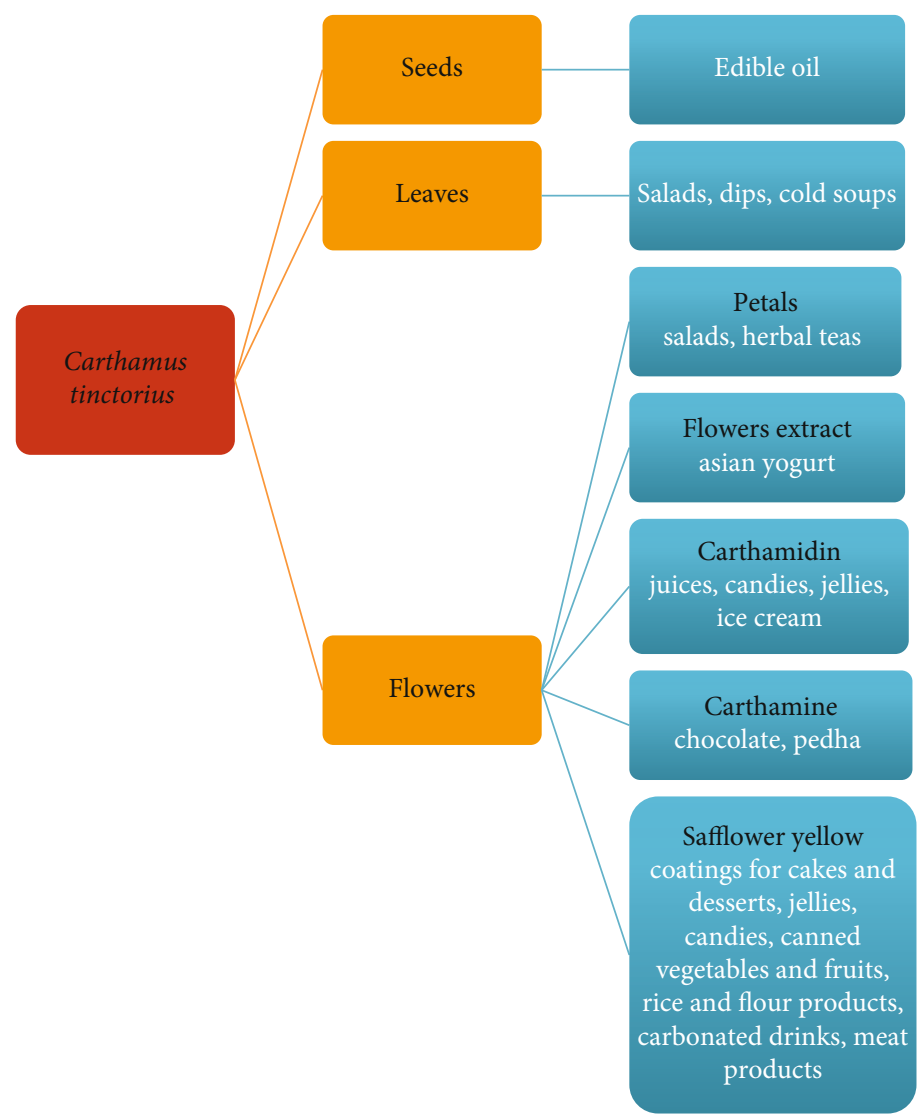

Figure 2: The use of safflower flowers and its components in food production.

decrease in fat and lactose content, as well as acidity. The percentage sucrose content when $5 \%$ addition carthamine was used was slightly lower than in the product without the pigment, but higher when the dose constituted $10 \%$ of product's mass. The highest score of sensory assessment was given to the product containing $10 \%$ carthamine (evaluated color, taste, flavor, and texture). Concentrate obtained from safflower was also used to produce Asian-style yogurt, a product with the addition of lychees and elderberry extract (Figure 2) [114].

However, the dyes contained in the safflower flowers have not been used in food production to take advantage of their health-promoting nature. The exception is herbal teas containing whole dried flowers. Introduction of HSYA to food production would be highly beneficial from the consumers' standpoint. This flavonoid is widely applied in medicine, and its addition to food products at the production stage, naturally at doses lower than therapeutic, would greatly improve their health-promoting value. Such products would be of substantial significance for the reduction of oxidative stress within different tissues and organs, and they could exhibit prophylactic action towards cardiovascular diseases and neoplastic diseases, among others.

An additional aspect favoring the addition of HSYA to foods is its beneficial impact on the reduction of adipose tissue and body weight following oral administration associated with its effect on the composition of intestinal microorganisms and cellular metabolism [67], on the inhibition of formation of new adipose cells [68] and enhancement of liver function [50]. Physical properties of HSYA (density $1.9 \pm$ $0.1 \mathrm{~g} / \mathrm{cm}^{3}$, boiling point at $1015.8 \pm 65^{\circ} \mathrm{C}$ at $760 \mathrm{mmHg}$, and ignition point at $334.0 \pm 27.8^{\circ} \mathrm{C}$; after [115]) indicate that it is not difficult for technological application. The only problem for HSYA application in food production is its poor absorption from the gastrointestinal tract: the absolute absorption was only $1.2 \%$ [116]. However, studies have shown that in order to improve absorption of HSYA, it should be used in the form of a water-in-oil microemulsion: then, the availability of this pigment for the organism increases by $1937 \%$, and digestion of the microemulsion occurs thanks to pancreatic lipase (in this form up to $60 \%$ of the microemulsion is digested within 1 hour) [117]. This observation opens the possibilities of enriching food products with the suitable consistency with HSYA: i.e., spreads and vegetable or fruit pastes. The process of microencapsulation of ingredients desired in food is also a great opportunity for HSYA.

Furthermore, research conducted on rats provided evidence that after intravenous administration of HSYA the presence of metabolites of the pigment was found in blood plasma, bile, urine and faeces, but these were not toxic values [116]. It is also important to note that the pigment did not accumulate in the organism: it was excreted mainly in urine [118] and faeces, and the half-life of its metabolites in blood was only 6 hours. After this time, 90\% of HSYA dosage was eliminated from the organism [116]. 


\section{Conclusions}

The wide commercial use of safflower means that the demand for its flowers is steadily increasing. In China, 1800-2600 MT of flowers were produced annually at the beginning of the 21st century [119]. Due to the possibility of using almost all parts of the plant, the profit from the cultivation of this plant is continuously growing (Sawant et al. (2000) by [10]). The health-promoting applicability of safflower increases with the progressing knowledge on its chemical composition.

\section{Conflicts of Interest}

The authors declare that they have no conflicts of interest.

\section{References}

[1] H. Caballero-Ortega, R. Pereda-Miranda, L. Riverón-Negrete et al., "Chemical composition of saffron (Crocus sativus L.) from four countries," Acta Horticulturae, vol. 650, article 59, pp. 321-326, 2004.

[2] R. Srivastava, H. Ahmed, R. K. Dixit, and S. A. S. Dharamveer, "Crocus sativus L.: a comprehensive review," Pharmacognosy Reviews, vol. 4, no. 8, pp. 200-208, 2010.

[3] F. Zarinkamar, S. Tajik, and S. Soleimanpour, "Effects of altitude on anatomy and concentration of crocin, picrocrocin and safranal in Crocus sativus L," Australian Journal of Crop Science, vol. 5, no. 7, pp. 831-838, 2011.

[4] C. Ulbricht, J. Conquer, D. Costa et al., "An evidence-based systematic review of saffron (Crocus sativus) by the Natural Standard Research Collaboration," Journal of Dietary Supplements, vol. 8, no. 1, pp. 58-114, 2011.

[5] M. Mashmoul, A. Azlan, H. Khaza'ai, B. N. M. Yusof, and S. M. Noor, "Saffron: a natural potent antioxidant as a promising anti-obesity drug," Antioxidants, vol. 2, no. 4, pp. 293308, 2013.

[6] T. Abu-Izneida, A. Rauf, A. A. Khalil et al., "Nutritional and health beneficial properties of saffron (Crocus sativus L): a comprehensive review," Critical Reviews in Food Science and Nutrition, vol. 2020, pp. 1-24, 2020.

[7] M. A. Maggi, S. Bisti, and C. Picco, "Saffron: chemical composition and neuroprotective activity," Molecules, vol. 25, no. 23 , p. $5618,2020$.

[8] R. Kumar, V. Singh, K. Devi, M. Sharma, M. K. Singh, and P. S. Ahuja, "State of art of saffron (Crocus sativus L.) agronomy: a comprehensive review," Food Reviews International, vol. 25, no. 1, pp. 44-85, 2009.

[9] J. A. Fernandez, "Biology, biotechnology and biomedicine of saffron," Recent Research Developments in Plant Science, vol. 2, pp. 127-159, 2004.

[10] V. Singh and N. Nimbkar, "Safflower (Carthamus tinctorius L.)," in Genetic Resources, Chromosome Engineering, and Crop Improvement volume 4, R. J. Singh, Ed., CRC press, Boca Raton, 2007.

[11] V. Emongor, "Safflower (Carthamus tinctorius L.) the underutilized and neglected crop: a review," Asian Journal of Plant Sciences, vol. 9, no. 6, pp. 299-306, 2010.

[12] X. Zhou, L. Tang, Y. Xu, G. Zhou, and Z. Wang, "Towards a better understanding of medicinal uses of Carthamus tinctorius L. in traditional Chinese medicine: a phytochemical and pharmacological review," Journal of Ethnopharmacology, vol. 151, no. 1, pp. 27-43, 2014.

[13] D. S. Hill, Pests of Crops in Warmer Climates and Their Control, Springer Science + Business Media, Dordrecht, 2008.

[14] M. D. Kaya, A. İpek, and A. Öztürk, "Effects of different soil salinity levels on germination and seedling growth of safflower (Carthamus tinctorius L.)," Turkish Journal of Agriculture and Forestry, vol. 27, pp. 221-227, 2003.

[15] R. Shabana, A. A. Abd El-Mohsen, H. A. H. Gouda, and H. S. Hafez, "Impact of temperature fluctuation on yield and quality traits of different safflower genotypes," Scientific Research and Review Journal, vol. 1, no. 3, pp. 74-87, 2013.

[16] A. Shirwaikar, S. Khan, Y. H. Kamariya, B. D. Patel, and F. P. Gajera, "Medicinal plants for the management of postmenopausal osteoporosis: a review," The Open Bone Journal, vol. 2, no. 1, pp. 1-13, 2010.

[17] X. Hu, S. Yin, Z. Huang, A. Elomri, and Y. Lu, "A new phenylpropanoid derivative isolated from Carthamus tinctorius L," Records of Natural Products, vol. 10, no. 1, pp. 17-21, 2016.

[18] M. Mohammadi, K. Ghassemi-Golezani, M. R. Chaichi, and S. Safikhani, "Seed oil accumulation and yield of safflower affected by water supply and harvest time," Agronomy Journal, vol. 110, no. 2, pp. 586-593, 2018.

[19] M. C. Sampaio, R. F. Santos, P. S. R. de Oliveira et al., "Comparison of safflower cultivation in two seasons in the south of Brazil," Journal of Agricultural Science, vol. 10, no. 5, pp. 92 99, 2018.

[20] R. F. Santos, D. Bassegio, M. M. P. Sartori, M. D. Zannoto, and M. A. Silva, "Safflower (Carthamus tinctorius L.) yield as affected by nitrogen fertilization and different water regimes," Acta Agronómica, vol. 67, no. 2, pp. 264-269, 2018.

[21] R. F. Santos, D. Bassegio, M. A. Silva, A. E. Klar, A. A. F. Silva, and T. R. B. Silva, "Irrigated safflower in phenological stages of Brazilian southeast dry season," IRRIGA, vol. 23, no. 3, pp. 493-504, 2018.

[22] C. Ren, J. Wang, B. Xian et al., "Transcriptome analysis of flavonoid biosynthesis in safflower flowers grown under different light intensities,” PeerJ, vol. 8, p. e8671, 2020.

[23] B. Coşge, B. Gürbüz, and M. Kiralan, "Oil content and fatty acid composition of some safflower (Carthamus tinctorius L.) varieties sown in spring and winter," International Journal of Natural and Engineering Sciences, vol. 1, no. 3, pp. 11-15, 2007.

[24] V. Vorpsi, F. Harizaj, N. Bardhi, V. Vladi, and E. Dodona, "Carthamus tinctorius L., the quality of safflower seeds cultivated in Albania," Research Journal of Agricultural Science, vol. 42, no. 1, pp. 326-331, 2010.

[25] K. Houmanat, H. Mazouz, M. El Fechtali, and A. Nabloussi, "Evaluation and pooling of safflower (Carthamus tinctorius L.) accessions from different world origins using agromorphological traits," International Journal of Advanced Research, vol. 5, no. 7, pp. 926-934, 2017.

[26] P. Golkar, "Inheritance of carthamin and carthamidin in safflower (Carthamus tinctorius L.)," Journal of Genetics, vol. 97, no. 1, pp. 331-336, 2018.

[27] S. La Bella, T. Tuttolomondo, L. Lazzeri, R. Matteo, C. Leto, and M. Licata, "An agronomic evaluation of new safflower (Carthamus tinctorius L.) germplasm for seed and oil yields under Mediterranean climate conditions," Agronomy, vol. 9, no. 8, p. $468,2019$. 
[28] Y. M. Liu, J. S. Yangand, and Q. H. Liu, "Studies on chemical constituents of Carthamus tinctorius," Chinese Traditional and Herbal Drugs, vol. 4, no. 28, pp. 288-289, 2005.

[29] F. Li, Z. He, and Y. Ye, "Isocartormin, a novel quinochalcone C -glycoside from Carthamus tinctorius," Acta Pharmaceutica Sinica B, vol. 7, no. 4, pp. 527-531, 2017.

[30] G. M. Machewad, P. Ghatge, V. Chappalwar, B. Jadhav, and A. Chappalwar, "Studies on extraction of safflower pigments and its utilization in ice cream," Journal of Food Processing \& Technology, vol. 3, no. 8, 2012.

[31] L.-L. Zhang, K. Tian, Z.-H. Tang et al., "Phytochemistry and pharmacology of Carthamus tinctorius L.," The American Journal of Chinese Medicine, vol. 44, no. 2, pp. 197-226, 2016.

[32] M. H. Cho, Y. S. Paik, and T. R. Hahn, "Enzymatic conversion of precarthamin to carthamin by a purified enzyme from the yellow petals of safflower," Journal of Agricultural and Food Chemistry, vol. 48, no. 9, pp. 3917-3921, 2000.

[33] L. Fan, H.-Y. Zhao, M. Xu et al., "Qualitative evaluation and quantitative determination of 10 major active components in Carthamus tinctorius L. by high-performance liquid chromatography coupled with diode array detector," Journal of Chromatography A, vol. 1216, no. 11, pp. 2063-2070, 2009.

[34] L. Fan, H. Y. Zhao, R. Pu, J. Han, B. R. Wang, and D. A. Guo, "Study on flavonoids in the flower of Carthamus tinctorius L," Journal of Chinese Pharmaceutical Sciences, vol. 46, no. 5, pp. 333-337, 2011.

[35] S. Yue, Y. Tang, S. Li, and J.-A. Duan, "Chemical and biological properties of quinochalcone C-glycosides from the florets of Carthamus tinctorius," Molecules, vol. 18, no. 12, pp. 15220-15254, 2013.

[36] V. A. Kurkin and A. V. Kharisova, "Flavonoids of Carthamus tinctorius flowers," Chemistry of Natural Compounds, vol. 50, no. 3, pp. 446-448, 2014.

[37] V. A. Kurkin, "Saffloroside, a new flavonoid from flowers of Carthamus tinctorius L," Journal of Pharmacognosy and Phytochemistry, vol. 4, no. 1, pp. 29-31, 2015.

[38] X. Xie, J. Zhou, L. Sun et al., "A new flavonol glycoside from the florets of Carthamus tinctorius L," Natural Product Research, vol. 30, no. 2, pp. 150-156, 2016.

[39] M. Xu, C. Du, N. Zhang, X. Shi, Z. Wu, and Y. Qiao, "Color spaces of safflower (Carthamus tinctorius L.) for quality assessment," Journal of Traditional Chinese Medical Sciences, vol. 3, no. 3, pp. 168-175, 2016.

[40] Z.-J. Pu, S.-J. Yue, G.-S. Zhou et al., "The comprehensive evaluation of safflowers in different producing areas by combined analysis of color, chemical compounds, and biological activity," Molecules, vol. 24, no. 18, p. 3381, 2019.

[41] A. A. Turgumbayeva, G. O. Ustenova, B. K. Yeskalieva et al., "Volatile oil composition of Carthamus tinctorius L. flowers grown in Kazakhstan," Annals of Agricultural and Environmental Medicine, vol. 25, no. 1, pp. 87-89, 2018.

[42] P. Ziarati, J. Asgarpanah, and M. Kianifard, “The essential oil composition of Carthamus tinctorius L. flowers growing in Iran," African Journal of Biotechnology, vol. 11, no. 65, pp. 12921-12924, 2012.

[43] S.-J. Yin, K.-Y. Liu, J. Lee et al., “Effect of hydroxysafflor yellow A on tyrosinase: integration of inhibition kinetics with computational simulation," Process Biochemistry, vol. 50, no. 12, pp. 2112-2120, 2015.

[44] Y. Zhao, H. Sun, X. Li, Y. Zha, and W. Hou, "Hydroxysafflor yellow A attenuates high glucose-induced pancreatic $\beta$-cells oxidative damage via inhibiting JNK/c-jun signaling pathway," Biochemical and Biophysical Research Communications, vol. 505, no. 2, pp. 353-359, 2018.

[45] C. Jin, J. Ying, W. Yang, and R. Liang, "Hydroxysafflor yellow A reverse chemotherapy resistance via JNK and P38 MAPK signal pathway in ovarian cancer cell," Farmácia, vol. 67, no. 2, pp. 331-336, 2019.

[46] M. Luo, J.-. C. Huang, Z.-. Q. Yang, Y.-. S. Wang, B. Guo, and Z.-. P. Yue, "Hydroxysafflor yellow A exerts beneficial effects by restoring hormone secretion and alleviating oxidative stress in polycystic ovary syndrome mice," Experimental Physiology, vol. 105, no. 2, pp. 282-292, 2019.

[47] D. Zhou, T. Ding, B. Ni, Y. Jing, and S. Liu, "Hydroxysafflor yellow A mitigated myocardial ischemia/reperfusion injury by inhibiting the activation of the JAK2/STAT1 pathway," International Journal of Molecular Medicine, vol. 44, pp. 405-416, 2019.

[48] T. Bacchetti, C. Morresi, L. Bellachioma, and G. Ferretti, "Antioxidant and pro-oxidant properties of Carthamus tinctorius, hydroxy safflor yellow A, and safflor yellow A," Antioxidants, vol. 9, no. 2, p. 119, 2020.

[49] M. Farid, S. S. Abdelgayed, M. H. Soliman, M. El-Fadhany, and R. H. Hussein, "Polyphenolic and flavonoids content, HPLC profiling and antioxidant activity of some medicinal plants with pancreatic histological study in alloxan-induced diabetic rats model," Journal of Microbiology, Biotechnology and Food Science, vol. 9, no. 4, pp. 746-750, 2020.

[50] K. Yan, X. Wang, H. Pan et al., "Safflower yellow and its main component HSYA alleviate diet-induced obesity in mice: possible involvement of the increased antioxidant enzymes in liver and adipose tissue," Frontiers in Pharmacology, vol. 11, p. $482,2020$.

[51] C. Qu, W. Zhu, K. Dong et al., "Inhibitory effect of hydroxysafflor yellow B on the proliferation of human breast cancer MCF-7 cells," Recent Patents on Anti-Cancer Drug Discovery, vol. 14, no. 2, pp. 187-197, 2019.

[52] S. Yue, Y. Tang, C. Xu, S. Li, Y. Zhu, and J. A. Duan, “Two new quinochalcone C-glycosides from the florets of Carthamus tinctorius," International Journal of Molecular Sciences, vol. 15, no. 9, pp. 16760-16771, 2014.

[53] E. Wincewicz, W. A. Zawadzki, and M. Ostaszewska, "Comparative study on the effect of evening primrose and safflower on selected morphological and biochemical blood parameters in experimental rats on a high-fat diet [in Polish]," Medycyna Weterynaryjna, vol. 71, no. 6, pp. 377-381, 2015.

[54] H. B. Nayaka, "Medicinal uses of selected wild edible vegetables consumed by Hyderabad-Karnataka region of Karnataka State in India," Asian Journal of Pharmacy and Pharmacology, vol. 6, no. 1, pp. 70-75, 2020.

[55] M. Ikram, A. M. Beshbishy, M. Kifayatullah et al., "Chemotherapeutic potential of Carthamus oxycantha root extract as antidiarrheal and in vitro antibacterial activities," Antibiotics, vol. 9, no. 5, p. 226, 2019.

[56] A.-r. Jo, H.-s. Han, S. Seo et al., "Inhibitory effect of moschamine isolated from Carthamus tinctorius on LPS-induced inflammatory mediators via AP-1 and STAT1/3 inactivation in RAW 264.7 macrophages," Bioorganic \& Medicinal Chemistry Letters, vol. 27, no. 23, pp. 5245-5251, 2017.

[57] R. Liu, T. Zhao, D. Che et al., "The anti-anaphylactoid effects of hydroxysafflor yellow A on the suppression of mast cell Ca2+ influx and degranulation," Phytomedicine, vol. 48, pp. 43-50, 2018. 
[58] W. Li, J. Liu, P. He et al., "Hydroxysafflor yellow A protects methylglyoxal-induced injury in the cultured human brain microvascular endothelial cells," Neuroscience Letters, vol. 549, pp. 146-150, 2013.

[59] M. Lee, H. Li, H. Zhao, M. Suo, and D. Liu, "Effects of hydroxysafflor yellow A on the PI3K/AKT pathway and apoptosis of pancreatic $\beta$-cells in type 2 diabetes mellitus rats," Diabetes, Metabolic Syndrome and Obesity: Targets and Therapy, vol. 13, pp. 1097-1107, 2020.

[60] Q. Li, F. Li, Z. Chen, S. Deng, X. Cao, and N. Tang, "Hydroxysafflor yellow A improves diabetes-induced renal fibrosis," Archives of Medical Science, vol. 16, no. 1, 2020.

[61] X.-D. Bie, J. Han, and H.-B. Dai, "Effects of hydroxysafflor yellow A on the experimental traumatic brain injury in rats," Journal of Asian Natural Products Research, vol. 12, no. 3, pp. 239-247, 2010.

[62] Y. Sun, D.-P. Xu, Z. Qin et al., "Protective cerebrovascular effects of hydroxysafflor yellow A (HSYA) on ischemic stroke," European Journal of Pharmacology, vol. 818, pp. 604-609, 2018.

[63] L. Tan, Y. Wang, Y. Jiang, R. Wang, J. Zu, and R. Tan, "Hydroxysafflor yellow A together with blood-brain barrier regulator lexiscan for cerebral ischemia reperfusion injury treatment," ACS Omega, vol. 5, no. 30, pp. 19151-19164, 2020.

[64] H. Xu, W. X. Liu, T. L. Liu et al., "Synergistic neuroprotective effects of Danshensu and hydroxysafflor yellow A on cerebral ischemia-reperfusion injury in rats," Oncotarget, vol. 70, no. 8, pp. 115434-115443, 2017.

[65] H. Li, Y. Liu, M. Wen et al., "Hydroxysafflor yellow A (HSYA) alleviates apoptosis and autophagy of neural stem cells induced by heat stress via p38 MAPK/MK2/Hsp27-78 signaling pathway," Biomedicine \& Pharmacotherapy, vol. 114, p. 108815, 2019.

[66] B. Han, L. Wang, F. Fu et al., "Hydroxysafflor yellow A promotes $\alpha$-synuclein clearance via regulating autophagy in rotenone-induced Parkinson's disease mice," Folia Neuropathologica, vol. 56, no. 2, pp. 133-140, 2018.

[67] J. Liu, S. Yue, Z. Yang et al., "Oral hydroxysafflor yellow A reduces obesity in mice by modulating the gut microbiota and serum metabolism," Pharmacological Research, vol. 134, pp. 40-50, 2018.

[68] Z. Hui-juan, W. Lin-jie, W. Xiang-qing et al., "Hormone-sensitive lipase is involved in the action of hydroxysafflor yellow A (HYSA) inhibiting adipogenesis of 3T3-L1cells," Fitoterapia, vol. 93, pp. 182-188, 2014.

[69] Z. Tang, H. Xie, S. Jiang et al., "Safflower yellow promotes angiogenesis through $\mathrm{p}$-VHL/HIF-1 $\alpha / \mathrm{VEGF}$ signaling pathway in the process of osteogenic differentiation," Biomedicine and Pharmacotherapy, vol. 107, pp. 1736-1743, 2018.

[70] D. Cui, D. Zhao, B. Wang et al., "Safflower (Carthamus tinctorius L.) polysaccharide attenuates cellular apoptosis in steroid-induced avascular necrosis of femoral head by targeting caspase-3-dependent signaling pathway," International Journal of Biological Macromolecules, vol. 116, pp. 106-112, 2018.

[71] D. Cui, D. Zhao, and S. Huang, "Beneficial contribution of a safflower (Carthamus tinctorius L.) polysaccharide on steroid-induced avascular necrosis of the femoral head in rats," International Journal of Biological Macromolecules, vol. 123, pp. 581-586, 2019.
[72] D. Cui, D. Zhao, and S. Huang, "Structural characterization of a safflower polysaccharide and its promotion effect on steroid-induced osteonecrosis in vivo," Carbohydrate Polymers, vol. 233, p. 115856, 2020.

[73] C. Wang, C. Wang, C. Ma et al., "Hydroxysafflor yellow A of Carthamus tinctorius attenuates lung injury of aged rats exposed to gasoline engine exhaust by down-regulating platelet activation," Phytomedicine, vol. 21, no. 3, pp. 199-206, 2014.

[74] X. Guo, M. Zheng, R. Pan, B. Zang, and M. Jin, "Hydroxysafflor yellow A suppresses platelet activating factor-induced activation of human small airway epithelial cells," Frontiers Pharmacology, vol. 9, p. 859, 2018.

[75] H. Ao, W. Feng, and C. Peng, "Hydroxysafflor yellow A: a promising therapeutic agent for a broad spectrum of diseases," Evidence-Based Complementary and Alternative Medicine, vol. 2018, Article ID 8259280, 17 pages, 2018.

[76] G. Yu, Z. Luo, Y. Zhou et al., "Uncovering the pharmacological mechanism of Carthamus tinctorius L. on cardiovascular disease by a systems pharmacology approach," Biomedicine \& Pharmacotherapy, vol. 117, p. 109094, 2019.

[77] D. Han, J. Wei, R. Zhang et al., "Hydroxysafflor yellow A alleviates myocardial ischemia/reperfusion in hyperlipidemic animals through the suppression of TLR4 signaling," Scientific Reports, vol. 6, no. 1, p. 35319, 2016.

[78] L. Li, P. Dong, C. Hou et al., "Hydroxysafflor yellow A (HSYA) attenuates hypoxic pulmonary arterial remodelling and reverses right ventricular hypertrophy in rats," Journal of Ethnopharmacology, vol. 186, pp. 224-233, 2016.

[79] J. Min and C. Wei, "Hydroxysafflor yellow A cardioprotection in ischemia-reperfusion (I/R) injury mainly via Akt/hexokinase II independent of ERK/GSK-3b pathway," Biomedicine \& Pharmacotherapy, vol. 87, pp. 419-426, 2017.

[80] X. Wang, Z. Zhou, Y. Xiong et al., "The mechanism of carthamin yellow injection promote acute myocardial infarction infarct border zone angiogenesis," in Proceedings of the 2016 International Conference on Biomedical and Biological Engineering, pp. 337-344, 2016.

[81] J. Yang, R. Wang, X. Cheng et al., "The vascular dilatation induced by hydroxysafflor yellow A (HSYA) on rat mesenteric artery through TRPV4-dependent calcium influx in endothelial cells," Journal of Ethnopharmacology, vol. 256, p. 112790, 2020.

[82] Y. Bai, P. Lu, C. Han et al., "Hydroxysafflor yellow A (HSYA) from flowers of Carthamus tinctorius L. and its vasodilatation effects on pulmonary artery," Molecules, vol. 17, no. 12, pp. 14918-14927, 2012.

[83] H.-X. Li, S.-Y. Han, X.-W. Wang et al., "Effect of the carthamins yellow from Carthamus tinctorius L. on hemorheological disorders of blood stasis in rats," Food and Chemical Toxicology, vol. 47, no. 8, pp. 1797-1802, 2009.

[84] C.-C. Li, C.-Z. Yang, X.-M. Li et al., "Hydroxysafflor yellow A induces apoptosis in activated hepatic stellate cells through ERK1/2 pathway in vitro," European Journal of Pharmaceutical Sciences, vol. 46, no. 5, pp. 397-404, 2012.

[85] C. Y. Wang, Q. Liu, Q. X. Huang et al., "Activation of PPAR $\gamma$ is required for hydroxysafflor yellow A of Carthamus tinctorius to attenuate hepatic fibrosis induced by oxidative stress," Phytomedicine, vol. 20, no. 7, pp. 592-599, 2013.

[86] F. Min, H. Sun, B. Wang et al., "Hepatoprotective effects of hydroxysafflor yellow A in D-galactose-treated aging mice," European Journal of Pharmacology, vol. 881, p. 173214, 2020. 
[87] R. Pant, R. Irchhaiya, and C. V. Rao, “A research article phytochemical and pharmacological evaluation of Carthamus tinctorius leaves," Journal of Drug Delivery and Therapeutics, vol. 9, no. 4-s, pp. 285-294, 2019.

[88] J.-p. Li, J.-m. Guo, Y.-q. Hua et al., "The mixture of Salvia miltiorrhiza-Carthamus tinctorius (Danhong injection) alleviates low-dose aspirin induced gastric mucosal damage in rats," Phytomedicine, vol. 23, no. 6, pp. 662-671, 2016.

[89] F. Yang, J. Li, J. Zhu, D. Wang, S. Chen, and X. Bai, "Hydroxysafflor yellow A inhibits angiogenesis of hepatocellular carcinoma via blocking ERK/MAPK and NF- $\kappa \mathrm{B}$ signaling pathway in H22 tumor-bearing mice," European Journal of Pharmacology, vol. 754, pp. 105-114, 2015.

[90] Y.-C. Ma, M.-M. Li, Q. Wu et al., "Hydroxysafflor yellow A sensitizes ovarian cancer cells to chemotherapeutic agent by decreasing WSB1 expression," European Journal of Integrative Medicine, vol. 25, pp. 6-12, 2019.

[91] J. Zhang, J. Li, H. Song, Y. Xiong, D. Liu, and X. Bai, "Hydroxysafflor yellow A suppresses angiogenesis of hepatocellular carcinoma through inhibition of p38 MAPK phosphorylation," Biomedicine \& Pharmacotherapy, vol. 109, pp. 806814, 2019.

[92] X. Chen, Y. Wang, L. Zhang, and Y. Gao, "Hydroxysafflor yellow A of Carthamus tinctorius L., represses the malignant development of esophageal cancer cells via regulating NF- $\kappa \mathrm{B}$ signaling pathway," Cell Biochemistry and Biophysics, vol. 78, no. 4, pp. 511-520, 2020.

[93] X. Liang and X.-Y. Hu, "The efficacy and safety of LDF, a Chinese herbal formula, compared with sorafenib for the treatment of advanced hepatocellular carcinoma in Chinese patients: a retrospective cohort study," International Journl of Clinical and Experimental Medicine, vol. 13, no. 1, pp. 126-133, 2020.

[94] Z. Chen, L. Liu, Y. Liu et al., "Hydroxysafflor yellow A induces autophagy in human liver cancer cells by regulating Beclin 1 and ERK expression," Experimental and Therapeutic Medicine, vol. 19, pp. 2989-2996, 2020.

[95] S. C. Wu, Y. Yue, H. Tian et al., "Carthamus red from Carthamus tinctorius L. exerts antioxidant and hepatoprotective effect against CCl4-induced liver damage in rats via the Nrf2 pathway," Journal of Ethnopharmacology, vol. 148, no. 2, pp. 570-578, 2013.

[96] S. Bahmanpour, Z. Vojdani, M. R. Panjehshahin, H. Hoballah, and H. Kassas, "Effects of Carthamus tinctorius on semen quality and gonadal hormone levels in partially sterile male rats," Korean Journal of Urology, vol. 53, no. 10, pp. 705-710, 2012.

[97] M. Mirhoseini, M. Mohamadpour, and L. Khorsandi, “Toxic effects of Carthamus tinctorius L. (safflower) extract on mouse spermatogenesis," Journal of Assisted Reproduction and Genetics, vol. 29, no. 5, pp. 457-461, 2012.

[98] Y.Z. Deng, "The adverse reaction of safflower injection in the clinical application," Strait Pharmaceutical Journal, vol. 24, pp. 228-230, 2012.

[99] Y. B. Zhang, H. Y. Dong, X. M. Zhao et al., "Hydroxysafflor yellow A attenuates carbon tetrachloride-induced hepatic fibrosis in rats by inhibiting Erk5 signaling," The American Journal of Chinese Medicine, vol. 40, no. 3, pp. 481-494, 2012.

[100] L. Zhang, Y. H. Yue, Y. C. Chen, G. Z. Yang, and X. R. Li, "Study on the type I allergic reaction of safflower injection," Chinese Journal of Hospital Pharmacy, vol. 17, pp. 13191321, 2012.
[101] Z. F. Liu, C. M. Li, M. Li, D. L. Li, and K. Liu, “The subchronic toxicity of hydroxysafflor yellow A of 90 days repeatedly intraperitoneal injections in rats," Toxicology, vol. 203, no. 1-3, pp. 139-143, 2004.

[102] Y. J. Choi, W. Sim, H. K. Choi, S. H. Lee, and B. H. Lee, " $\alpha$-Terpineol induces fatty liver in mice mediated by the AMP-activated kinase and sterol response element binding protein pathway," Food and Chemical Toxicology, vol. 55, pp. 129-136, 2013.

[103] T. Watanabe, N. Hasegawa, A. Yamamoto, S. Nagai, and S. Terabe, "Separation and determination of yellow and red safflower pigments in food by capillary electrophoresis," Bioscience, Biotechnology and Biochemistry, vol. 61, pp. 11791183, 1997.

[104] P. P. Danieli, R. Primi, B. Ronchi et al., "The potential role of spineless safflower (Carthamus tinctorius L. var. inermis) as fodder crop in central Italy," Italian Journal of Agronomy, vol. 6, no. 1, p. 4, 2011.

[105] N. Fatahi, J. Carapetian, and R. Heidari, "Spectrophotometric measurement of valuable pigments from petals of safflower (Carthamus tinctorius L.) and their identification by TLC metod," Research Journal of Biological Sciences, vol. 3, no. 7, pp. 761-763, 2008.

[106] E. Delshad, M. Yousefi, P. Sasannezhad, H. Rakhshandeh, and Z. Ayati, "Medical uses of Carthamus tinctorius L. (safflower): a comprehensive review from traditional medicine to modern medicine," Electronic Physician, vol. 10, no. 4, pp. 6672-6681, 2018.

[107] P. G. Peiretti, "Nutritional aspects and potential uses of safflower (Carthamus tinctorius L.) in livestock," in Agricultural research updates, Nova science publishers Inc, 2017, https:// www.researchgate.net/publication/315517724_Nutritional_ aspects_and_potential_uses_of_safflower_Carthamus_ tinctorius_L_in_livestock/link/58d38e06a6fdccd24d43d846/ download.

[108] H. H. Mundel, R. E. Blackshaw, J. R. Byers et al., "Safflower production on the Canadian prairies: revisited in 2004," in Agriculture and Agri-Food Canada, p. 37, Lethbridge Research Centre, Lethbridge, Alberta, 2004.

[109] Y. Dai, R. Verpoorte, and Y. H. Choi, "Natural deep eutectic solvents providing enhanced stability of natural colorants from safflower (Carthamus tinctorius)," Food Chemistry, vol. 159, pp. 116-121, 2014.

[110] Y. H. Choi, J. van Spronsen, Y. Dai et al., “Are natural deep eutectic solvents the missing link in understanding cellular metabolism and physiology?," Plant Physiology, vol. 156, no. 4, pp. 1701-1705, 2011.

[111] S. Lehto, M. Buchweitz, A. Klimm, R. Strassburger, C. Bechtold, and F. Ulberth, "Comparison of food colour regulations in the EU and the US: a review of current provisions," Food Additives \& Contaminants: Part A, vol. 34, no. 3, pp. 335-355, 2017.

[112] "A global company specializing in the production phycocuanin "Binmei Biotechnology"," 2020, http://www.binmeibio .com/.

[113] G. M. Machewad, B. A. Jadhav, A. G. Lamdande, and P. V. Wadkar, "Extraction of safflower yellow pigment (Carthimidin) and its fortification in Pedha," Food Science Research Journal, vol. 4, no. 1, pp. 46-48, 2013.

[114] K. Szwedziak and D. Antczak, "Zdrowotne właściwości jogurtów smakowych,” Postępy Techniki Przetwórstwa Spożywczego, vol. 1, pp. 19-21, 2016. 
[115] C. S. A. R. Reserved, A Smart Chem-Search Engine, 2018, https://www.chemsrc.com/en/.

[116] Y. Jin, L. Wu, Y. Tang et al., "UFLC-Q-TOF/MS based screening and identification of the metabolites in plasma, bile, urine and feces of normal and blood stasis rats after oral administration of hydroxysafflor yellow A," Journal of Chromatography B, vol. 1012-1013, pp. 124-129, 2016.

[117] J. Qi, J. Zhuang, W. Wu et al., "Enhanced effect and mechanism of water-in-oil microemulsion as an oral delivery system of hydroxysafflor yellow A," International Journal of Nanomedicine, vol. 6, pp. 985-991, 2011.

[118] C.-Y. Li, J.-G. Yin, J. Zhang et al., "Pharmacokinetic profiles of hydroxysafflor yellow A following intravenous administration of its pure preparations in healthy Chinese volunteers," Journal of Ethnopharmacology, vol. 162, pp. 225-230, 2015.

[119] W. Zhaomu and D. Lijie, "Current situation and prospects of safflower products development in China," in Proceedings of the Fifth International Safflower Conference, pp. 315-319, Williston, North Dakota and Sidney, USA, 2001.

[120] ChemSpider Search and share chemistry, Royal Society of Chemistry, 2021, http://www.chemspider.com/.

[121] PubChem, National Center for Biotechnology Information, National Library of Medicine, USA, 2021, https://pubchem .ncbi.nlm.nih.gov/.

[122] M. Yang, S. Tao, S. Guan, X. Wu, P. Xu, and D. Guo, "Chinese traditional medicine," in Comprehensive Natural Products II, pp. 383-477, Chemistry and Biology 3, 2010. 